Article

\title{
Assessment of Density-Functional Tight-Binding Ionization Potentials and Electron Affinities of Molecules of Interest for Organic Solar Cells Against First-Principles $G W$ Calculations
}

\author{
Ala Aldin M. H. M. Darghouth ${ }^{1,7}$, Mark E. Casida ${ }^{1, *}$, Walid Taouali ${ }^{2}$, Kamel Alimi ${ }^{2}$, \\ Mathias P. Ljungberg ${ }^{3,4}$, Peter Koval ${ }^{4,5}$, Daniel Sánchez-Portal ${ }^{4,5}$ and Dietrich Foerster ${ }^{6}$ \\ ${ }^{1}$ Département de Chimie Moléculaire (DCM, UMR CNRS/UJF 5250), Institut de Chimie Moléculaire \\ de Grenoble (ICMG, FR2607), Université Joseph Fourier (Grenoble I), 301 rue de la Chimie, BP 53, \\ F-38041 Grenoble Cedex 9, France; E-Mail: ala.darghouth@ujf-grenoble.fr \\ ${ }^{2}$ Unité de Recherche: Matériaux Nouveaux et Dispositifs Électroniques Organiques (UR 11ES55), \\ Faculté des Sciences de Monastir, Université de Monastir, 5000 Monastir, Tunisia; \\ E-Mails: tawali_walid@yahoo.fr(W.T.); kamealimi@yahoo.fr (K.A.) \\ ${ }^{3}$ Department of Physics, Phillips-University Marburg, Renthof 5, 35032 Marburg, Germany; \\ E-Mail: mathias.ljungberg@gmail.com \\ ${ }^{4}$ Donostia International Physics Center (DIPC), Paseo Manuel de Lardizabal 4, \\ E-20018 San Sebastián, Spain; E-Mails: koval.peter@gmail.com (P. K.); sqbsapod@ehu.eus (D.S.-P.) \\ ${ }^{5}$ Centro de Física de Materiales CFM-MPC, Centro Mixto CSIC-UPV/EHU, Paseo Manuel de \\ Lardizabal 5, E-20018 San Sebastián, Spain \\ ${ }^{6}$ CPMOH/LOMA, Université de Bordeaux 1, 351 Cours de la Liberation, 33405 Talence, France; \\ E-Mail: dietrich.foerster@u-bordeaux.fr \\ ${ }^{7}$ Department of Chemistry, College of Science, University of Mosul, Mosul 41002, Iraq \\ * Author to whom correspondence should be addressed; E-Mail: mark.casida@ujf-grenoble.fr; \\ Tel.: +33-04-76-63-56-28.
}

Academic Editors: Karlheinz Schwarz and Agnes Nagy

Received: 14 August 2015 / Accepted: 19 November 2015 / Published: 4 December 2015

\begin{abstract}
Ionization potentials (IPs) and electron affinities (EAs) are important quantities input into most models for calculating the open-circuit voltage $\left(V_{o c}\right)$ of organic solar cells. We assess the semi-empirical density-functional tight-binding (DFTB) method with the third-order self-consistent charge (SCC) correction and the 3ob parameter set (the third-order DFTB (DFTB3) organic and biochemistry parameter set) against experiments (for smaller molecules) and against first-principles $G W$ (Green's function, G, times the
\end{abstract}


screened potential, W) calculations (for larger molecules of interest in organic electronics) for the calculation of IPs and EAs. Since $G W$ calculations are relatively new for molecules of this size, we have also taken care to validate these calculations against experiments. As expected, DFTB is found to behave very much like density-functional theory (DFT), but with some loss of accuracy in predicting IPs and EAs. For small molecules, the best results were found with $\triangle \mathrm{SCF}$ ( $\Delta$ self-consistent field) SCC-DFTB calculations for first IPs (good to $\pm 0.649 \mathrm{eV}$ ). When considering several IPs of the same molecule, it is convenient to use the negative of the orbital energies (which we refer to as Koopmans' theorem (KT) IPs) as an indication of trends. Linear regression analysis shows that KT SCC-DFTB IPs are nearly as accurate as $\triangle$ SCF SCC-DFTB eigenvalues ( $\pm 0.852 \mathrm{eV}$ for first IPs, but $\pm 0.706 \mathrm{eV}$ for all of the IPs considered here) for small molecules. For larger molecules, SCC-DFTB was also the ideal choice with IP/EA errors of $\pm 0.489 / 0.740 \mathrm{eV}$ from $\triangle \mathrm{SCF}$ calculations and of $\pm 0.326 / 0.458 \mathrm{eV}$ from (KT) orbital energies. Interestingly, the linear least squares fit for the KT IPs of the larger molecules also proves to have good predictive value for the lower energy KT IPs of smaller molecules, with significant deviations appearing only for IPs of 15-20 eV or larger. We believe that this quantitative analysis of errors in SCC-DFTB IPs and EAs may be of interest to other researchers interested in DFTB investigation of large and complex problems, such as those encountered in organic electronics.

Keywords: organic solar cells; ionization potentials; electron affinities; density-functional theory (DFT); density-functional tight-binding (DFTB); Green's functions; $G W$

\section{Introduction}

Organic electronics is a rapidly-growing alternative to silicon-based electronics. In contrast to the latter, which is well enough understood [1], that fabless manufacturing may be used for circuit design using modeling software, such as SPICE [2], and then be outsourced to a semiconductor foundry for actual fabrication, organic electronics is a rapidly growing, but much less well understood [3-8] technology. One example is organic solar cells [9-12], which offer the advantage of being relatively inexpensive to manufacture, flexible and printable. The problem then is to be able to understand such devices well enough to be able to optimize and ultimately to engineer devices with them. This implies modeling, and modeling of organic solar cells almost always requires ionization potentials (IPs) and electron affinities (EAs) as input. However, the size of the molecules (or clusters of molecules) used in modeling organic solar cells rapidly makes first-principles calculations prohibitive, if not impossible. The semi-empirical density-functional tight-binding (DFTB) method is highly attractive for overcoming size and complexity modeling constraints, but it needs to be carefully assessed to determine the expected accuracy. In this paper, we complement previous efforts [13,14] ([14] concerns implementing range-separated hybrids in DFTB $[15,16]$ ) by assessing DFTB against accurate first-principles $G W$ (Green's function, G, times the screened potential, W) calculations [17-19] for the calculation of the IPs and EAs of medium-sized molecules. 
Our particular interest is in modeling organic solar cells for which phenomenological models are reviewed in the Appendix. Ideally, we would like to be able to extract parameters for these models from atomistic modeling. Given the size and complexity of minimum-sized cluster models or unit cells of periodic models for a reasonable description of organic solar cells, it is clear that we need a highly efficient method. This is especially clear for such things as charge diffusion lengths and the size of depletion zones. However, this is also true for the seemingly relatively straightforward calculation of IPs and EAs. Figure 1 gives minimum size examples of some typical molecules that are used in organic electronics. While some are "medium-sized" molecules, which come within the range of ab initio calculations, many of the examples shown are just oligomers intended to represent the much longer polymers used in real organic solar cells. To make matters worse, charge transfer excitons are often present, which are delocalized over several "medium-sized" molecules, so that it is necessary to use clusters of molecules to have a physically-reasonable model. All of these reasons encourage us to look for an efficient and hopefully reasonably accurate semi-empirical approach. An appealing approach that may meet our needs is the density-functional tight-binding (DFTB) approach, which will be briefly reviewed in the next section. To our knowledge, there is only one previous application of DFTB to organic solar cells [13]. That study focused on the problem of calculating charge transfer energies and made a comparison against experimental values.

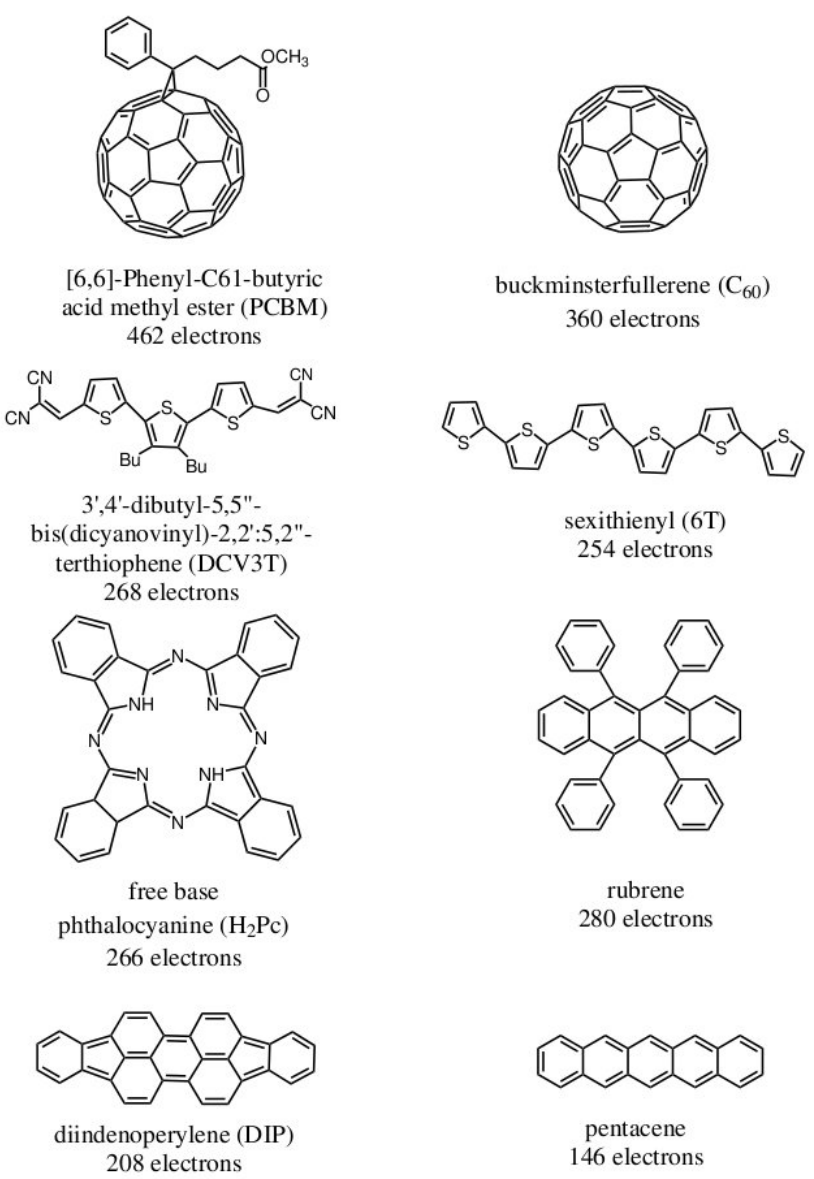

Figure 1. Eight typical molecules that are often used as acceptors or donors in organic electronics. 
We have chosen a different approach to assessing the accuracy of DFTB here by choosing to compare DFTB IPs and EAs against first-principles $G W$ values. The advantage of this approach is that experimental IPs and EAs for large molecules are often extracted from condensed phase (bulk, thin film or solution) measurements, introducing uncertainties when it comes to trying to extract molecular values. We will show that our $G W$ calculations do indeed give good IPs when compared to experimental IPs for medium-sized molecules, and we will then assume that this is also true for the other medium-sized molecules, where molecular IPs are not available and also for EAs. Taking the $G W$ values as the "true values", we then calculate error bars on the DFTB estimate of the $G W$ values.

The rest of this paper is organized as follows: the next section gives a brief review of the basic electronic structure methods used in this paper. Section 3 provides computational details. Section 4 goes on to describe and apply our analytical procedure for assessing the quality of DFTB IPs and EAs for the case of small molecules where good and theoretically well-understood experimental data are available. This gives us a useful first indication of how DFTB might work for the case that really interests us, namely for larger molecules of interest in organic electronics. Section 5 then extends our analysis to a set of medium-sized molecules typical of organic electronics. The assessment is complicated by the dearth of theoretically well-understood experimental data. We show that this dearth of experimental data may be compensated by high-quality $G W$ calculations, which agree well with available experimental data, and best when for gas phase data, where the comparison is cleanest. We therefore use the $G W$ results as our benchmark for evaluating DFTB IPs and EAs for medium-sized molecules. Section 6 provides a concluding discussion.

\section{Theoretical Methods}

While the Hartree-Fock (HF) approximation and density-functional theory (DFT) are now so familiar that they hardly seem worth discussing, this is less the case for $G W$ Green's function calculations and for the DFTB semiempirical approximation to DFT (HF is treated in most text books on quantum chemistry, such as [20], while DFT is treated in many recent text books; nevertheless more detailed information regarding DFT may be found in [21-25]). These latter approaches will be briefly described in this section, with emphasis on calculating IPs and EAs. Unless otherwise specified, we will use Hartree atomic units $\left(\hbar=m_{e}=e=1\right)$. We will also use a reduced notation where $q=\left(\mathbf{r}_{q}, \sigma_{q}\right)$ represents the space and spin coordinates of particle $q$ while $\mathbf{q}=\left(q, t_{q}\right)$ (bold face) is enriched to include time.

All approaches involve a molecular orbital (MO) equation,

$$
\hat{f}\left(1 ; \epsilon_{s}\right) \psi_{s}(1)=\epsilon_{s} \psi_{s}(1)
$$

The MO Hamiltonian has the form,

$$
\hat{f}(1 ; \omega)=\hat{h}_{H}(1)+\hat{\Sigma}_{x c}(1, \omega)
$$

where $\hat{h}_{H}$ is the Hartree Hamiltonian. The frequency-dependence of the self-energy belongs to the $G W$ method and does not apply for the HF approximation nor for Kohn-Sham DFT. The exchange-correlation (xc) "self-energy", $\hat{\Sigma}_{x c}(1 ; \omega)$, is just the exchange integral operator $[-\hat{K}(1)]$ in HF and the functional derivative $v_{x c}(1)=\delta E_{x c} / \delta \rho(1)$ in pure DFT (we use $\rho$ for the density). The MO equation is obtained 
in both cases from the variational minimization of the corresponding energy expressions. It is now also common practice to mix HF and pure DFT to make hybrid functionals, such as the popular B3LYP functional.

One way to calculate the (first) IP, $I$, and (first) EA, $A$, in DFT is to carry out self-consistent field calculations of the total energies for the $N$-electron system, the $(N-1)$-electron system and the $(N+1)$-electron system. The $\Delta \mathrm{SCF}$ ( $\Delta$ self-consistent field) IPs and EAs are then given respectively by,

$$
\begin{aligned}
I_{S} & =E_{S}^{(N-1)}-E_{0}^{(N)} \\
A_{S} & =E_{0}^{(N)}-E_{S}^{(N+1)}
\end{aligned}
$$

where $E_{0}^{(N)}$ is the energy of the ground state of the $N$-electron molecule, $E_{S}^{(N-1)}$ is the energy of the same system with one electron removed in its $S$-th electronic state and $E_{S}^{(N+1)}$ is the energy of the same system, but with one more electron in its $S$-th excited state. Note that the concepts of IP and EA have thus been generalized beyond the usual definition where $S=0$ (ground state). Together, the IPs and EAs form a (generalized) band spectrum. Note also that $S$ is a state label, as opposed to an MO label, which would have been a small $s$. In the quasiparticle part of the band spectrum, each principle IP or EA corresponds to removing or adding an electron to an orbital $s$. In this case, $S$ may be identified with $s$. The $\triangle$ SCF IPs and EAs may be estimated using Slater's transition orbital method, whereby the $\triangle \mathrm{SCF}$ IP or EA is given by minus the orbital energy recalculated with half an electron removed from or added to the orbital. The $\triangle \mathrm{SCF}$ method works for small molecules, but must inevitably fail for pure DFT (where the density functional depends only on the density as in the local density approximation (LDA) or generalized gradient approximations (GGAs)) in the limit of a large system with delocalized hole and electron states, because the change in the charge density is then insignificant [26]. Furthermore, the $\triangle \mathrm{SCF}$ method is known to fail to give good IPs and EAs in the HF approximation, because it includes only relaxation effects and not correlation effects, which are known to partially cancel each other for outer valence ionization. In this case, it is better to use Koopmans' theorem IPs and EAs, which are just the negative of the orbital energies. Such IPs and EAs are still approximate.

\subsection{Green's Function Methods}

Green's function methods may be thought of as adding terms to the xc self-energy, so that Koopmans' theorem IPs and EAs become exact. That is, minus the energies of the occupied orbitals are the vertical IPs, and minus the unoccupied orbital energies are the vertical EAs. More exactly, the MO equation has two types of solutions, namely the IP solutions and the EA solutions. For the IP solutions,

$$
\begin{aligned}
\epsilon_{S} & =E_{0}^{(N)}-E_{S}^{(N-1)} \\
\psi_{S}(1) & =\left\langle\Psi_{S}^{(N-1)}|\hat{\psi}(1)| \Psi_{0}^{(N)}\right\rangle \\
& =\sqrt{N} \int\left(\Psi_{S}^{(N-1)}(2,3, \cdots, N)\right)^{*} \\
& \times \Psi_{0}^{(N)}(1,2,3, \cdots, N) d 2 d 3 \cdots d N
\end{aligned}
$$


while for the EA solutions,

$$
\begin{aligned}
\epsilon_{S} & =E_{S}^{(N+1)}-E_{0}^{(N)} \\
\psi_{S}(1) & =\left\langle\Psi_{0}^{(N)}|\hat{\psi}(1)| \Psi_{S}^{(N+1)}\right\rangle \\
& =\sqrt{N+1} \int\left(\Psi_{0}^{(N)}(2,3, \cdots, N+1)\right)^{*} \\
& \times \Psi_{S}^{(N+1)}(1,2,3, \cdots, N+1) d 2 d 3 \cdots d(N+1)
\end{aligned}
$$

where $\hat{\psi}(1)$ is an annihilation field operator in second-quantized notation, $\Psi_{0}^{(N)}$ is the ground-state $N$-electron wave function; $\Psi_{S}^{(N-1)}$ is the $S$-th excited state of the $(N-1)$-electron system; and $\Psi_{S}^{(N+1)}$ is the $S$-th excited state of the $(N+1)$-electron system. Note the use of capital indices referring to states rather than orbitals, since there can be several different IP (or EA) states corresponding to the same orbital due to many-body effects. The MOs are then more properly described as Dyson amplitudes or as ion-molecule overlaps (both names are used). However, for outer-valence ionization and the first few electron attachment states (i.e., in the so-called "quasiparticle regime"), a single state usually dominates, and we may use lower case (i.e., orbital) notation. The definition of the Dyson amplitudes implies that they are not normalized to unity $\left(\left\langle\Psi_{S} \mid \Psi_{S}\right\rangle=P_{S} \neq 1\right)$. The correct normalization ("pole strength") of the MOs is obtained by:

$$
\left\langle\psi_{S} \mid \psi_{S}\right\rangle=\left[1-\left.\frac{\partial}{\partial \omega} \Re \frac{\left\langle\psi_{S}\left|\hat{\Sigma}_{x c}(\omega)\right| \psi_{S}\right\rangle}{\left\langle\psi_{S} \mid \psi_{S}\right\rangle}\right|_{\omega=\epsilon_{S}}\right]^{-1}
$$

Note that any choice of normalization factor for MO may be used on the right-hand side of the equation, as the normalization factor cancels out on that side.

So far, Green's function is exact, but also computationally useless until self-energy approximations are introduced. This is usually done in quantum chemistry by taking the HF approximation as the zero-order picture and expanding the self-energy in a perturbation series in the fluctuation potential (i.e., in the difference between the bare interelectronic coulomb repulsion and the HF self-consistent field). The lowest order corrections are second (GF2, second-order of Green's function) and third (GF3, third-order of Green's function) order, because the first-order correction is zero by Brillouin's theorem. However, order-by-order expansion of IPs and EAs typically leads at best to slow oscillatory convergence. In the outer-valence Green's function (OVGF) method, the GF2 and GF3 results are renormalized to obtain an infinite order estimate ([27] and Appendix $\mathrm{C}$ of [28]). This is the conventional approach for most molecular Green's function calculations. However, there is another approach, due to Hedin [17], which is based on an expansion in the dynamically-screened interaction, $W(\mathbf{1}, \mathbf{2})$. The self-energy is essentially given by the Green's function times the dynamically-screened interaction,

$$
\Sigma_{x c}(\mathbf{1}, \mathbf{2})=i G(\mathbf{1}, \mathbf{2}) W\left(\mathbf{1}^{+}, \mathbf{2}\right)
$$

As both $G$ and $W$ are constructed from the solutions of the MO equation, the $G W$ method should, in principle, be solved self-consistently. In practice, it is usually just applied in a one-step process $\left(G_{0} W_{0}\right.$ approximation) after a DFT calculation and is hence dependent on the functional used in the original DFT calculation. After some early struggles [29-35], the $G W$ approximation is also proving to be useful for 
molecules $[19,36]$. The main advantage over the older OVGF molecular approach may be computational efficiency. This is particularly important, as Green's function calculations require larger basis sets than either HF or DFT. In the case of HF, this is because of the neglect of electron correlation. In the case of DFT, this is because it is easier to describe the charge density than the correlated wave function. In our work, we take advantage of the efficiency of the dominant product algorithm for $G W$ calculations [19].

It is interesting to note that the xc potential of pure DFT may be regarded as the best local approximation to the nonlocal xc self-energy of Green's function theory [37] which is the basis of the popular target Kohn-Sham approximation in electron momentum spectroscopy [38-40]. In fact, experience has shown that Kohn-Sham orbitals are a reasonably good (apparently often better than HF) estimate of Dyson orbitals. Furthermore, while Kohn-Sham "Koopmans' theorem" IPs underestimate experimental IPs, they are in fact a better estimate than HF Koopmans' theorem IPs, just systematically down-shifted because of the particle number derivative discontinuity effect on the long-range behavior of the xc-potential [41]. This is the molecular analogue of the solid-state scissor's operator correction to band energies [42-44]. We will return to this point again in the next section.

\subsection{Density-Functional Tight-Binding}

Having given a brief, but hopefully adequately detailed discussion of the Green's function method, so as to keep this paper relatively self-contained, let us now turn our attention to a brief review of the semi-empirical DFTB approximation to DFT. Although tight-binding theory has its roots in work by Erich Hückel [45-47], Slater and Koster [48] and Roald Hoffmann [49-52], density-functional tight-binding (DFTB) [53-56] also includes elements of modern semi-empirical theory [57,58] and density-functional theory [59,60]. As we will continue to use the same notation, our notation will be somewhat different than that typically used in the DFTB literature.

DFTB began as a way to extract an extended Hückel or tight-binding-like approximation from DFT [53]. At the heart of DFTB is an assumption of separability, which is actually exact for both the LDA and for GGAs of the xc energy. In DFTB, we first separate the charge density into non-overlapping atomic parts,

$$
\rho(1)=\sum_{I} \rho_{I}(1)
$$

The sum is over atoms $I$. It follows that the Hartree $(\mathrm{H})$ potential is separable as,

$$
v_{H}[\rho](1)=\sum_{I} v_{H}\left[\rho_{I}\right](1)=\sum_{I} v_{H}^{I}(1)
$$

In the LDA and GGAs,

$$
\begin{aligned}
E_{x c}[\rho] & =\sum_{I} E_{x c}\left[\rho_{I}\right]=\sum_{I} E_{x c}^{I} \\
v_{x c}[\rho](1) & =\sum_{I} v_{x c}\left[\rho_{I}\right](1)=\sum_{I} v_{x c}^{I}(1)
\end{aligned}
$$

Hence, the potential part of the Kohn-Sham operator is separable,

$$
v_{s}(1)=\sum_{I} v_{I}(1)+\sum_{I} v_{H}^{I}(1)+\sum_{I} v_{x c}^{I}(1)
$$


We may now make typical approximations familiar in semi-empirical theory in order to avoid having to calculate more than two center integrals. Thus, if atomic orbitals (AOs) $\mu$ and $\nu$ are both on atom $I$, then the matrix elements of the DFTB orbital Hamiltonian:

$$
f_{\mu \in I, \nu \in I}=\delta_{\mu, \nu} \epsilon_{\mu}^{I}
$$

where $\epsilon_{\mu}^{I}$ is the $\mu$-th $\mathrm{AO}$ of the free atom $I$. For off-diagonal elements where $\mu$ is on atom $I$ and $\nu$ is on a different atom $J$, then,

$$
f_{\mu \in I, \nu \in J}=\left\langle\chi_{\mu \in I}\left|\left(\hat{t}_{s}+v_{s}^{I}+v_{s}^{J}\right)\right| \chi_{\nu \in J}\right\rangle
$$

where $\hat{t}=-(1 / 2) \nabla^{2}$. It is noteworthy that the "potential superposition approximation" of Equation (13) is replaced by the "density superposition approximation":

$$
f_{\mu \in I, \nu \in J}=\left\langle\chi_{\mu \in I}\left|\left(\hat{t}_{s}+v_{s}^{I, J}\right)\right| \chi_{\nu \in J}\right\rangle
$$

with:

$$
v_{s}^{I, J}=v_{I}+v_{J}+v_{H}\left[\rho_{I}+\rho_{J}\right]+v_{x c}\left[\rho_{I}+\rho_{J}\right]
$$

as significant differences in DFTB orbital energies may be expected between the two approaches and as the latter approach is that actually used in the DFTB program and parameters whose results are reported in this paper [14]. Whichever choice is used, the AOs $\chi_{\mu}$ are typically calculated for a slightly confined atom to emulate the effect of an atom in a molecule. The overlap matrix,

$$
s_{\mu \in I, \nu \in J}=\left\langle\chi_{\mu \in I} \mid \chi_{\mu \in J}\right\rangle
$$

is calculated exactly, as it only involves two center integrals. No self-consistent procedure is then needed to set up and solve the matrix form of the Kohn-Sham equation in the AO basis set,

$$
\mathbf{f} \vec{c}_{r}=\epsilon_{i} \mathbf{S} \vec{c}_{r}
$$

A very similar approximation is used to evaluate the so-called "repulsive energy",

$$
E_{r e p}=\sum_{I<J} G_{I, J}
$$

The pair energies $G_{I, J}\left(R_{I, J}\right)$ are obtained by fitting to a variation of $E_{r e p}$ as a function of the distance $R_{I, J}$ between atoms of the same types as $I$ and $J$ for a variety of molecules. This allows geometries to be optimized.

Notice that the original form of DFTB begins with the orbital Hamiltonian and derives the total energy from the orbital energies. However, it is important to know how to proceed the other way around, namely to know how to begin with the energy expression and derive the orbital Hamiltonian. We can do this by assuming the repulsive energy to be independent of the density matrix,

$$
\mathbf{P}=\sum_{r} \vec{c}_{r} n_{r} \vec{c}_{r}^{\dagger}
$$


and writing the so-called "band structure term" as,

$$
E_{B S}=\operatorname{tr}(\mathbf{P f})
$$

then,

$$
f_{\nu, \mu}=\frac{\partial E}{\partial P_{\mu, \nu}}=\frac{\partial E_{B S}}{\partial P_{\mu, \nu}}
$$

is obtained in the usual way from the method of Lagrange multipliers.

A few years after the introduction of the original DFTB scheme, a self-consistent charge (SCC) term was added to the DFTB energy expression in order to describe charge rearrangement of atoms within molecules $[61,62]$. In the original DFTB (DFTB2), the charge fluctuation term is only expanded up to second order in the variation of the atomic charge densities upon molecule formation. In recent years, the charge fluctuation term has been extended to third order to make DFTB3 [62]. We briefly describe the DFTB3 method here. The electronic energy expression thus gains what is known in DFTB as a charge fluctuation term $\left(E_{\text {coul }}\right)$, so that:

$$
\begin{gathered}
E=E_{B S}+E_{\text {rep }}+E_{\text {coul }} \\
E_{\text {coul }}=E_{\text {coul }}^{(2)}+E_{\text {coul }}^{(3)} \\
E_{\text {coul }}^{(2)}=\frac{1}{2} \iint f_{\text {Hxc }}(1,2) \delta \rho(1) \delta \rho(2) d 1 d 2 \\
E_{\text {coul }}^{(3)}=\frac{1}{6} \iiint g_{x c}(1,2,3) \delta \rho(1) \delta \rho(2) \delta \rho(3) d 1 d 2 d 3
\end{gathered}
$$

where the combined $\mathrm{H}$ and $\mathrm{xc}$ kernels are,

$$
\begin{aligned}
f_{H x c}(1,2) & =\frac{1}{r_{12}}+\frac{\delta E_{x c}}{\delta \rho(1) \delta \rho(2)} \\
g_{x c}(1,2,3) & =\frac{\delta^{2} E_{x c}}{\delta \rho(1) \delta \rho(2) \delta \rho(3)}
\end{aligned}
$$

By making use of the same separability assumptions as in the original DFTB, we arrive at,

$$
\begin{aligned}
& E_{\text {coul }}^{(2)}=\frac{1}{2} \sum_{I, J} \iint f_{H x c}^{I, J}(1,2) \delta \rho^{I}(1) \delta \rho^{J}(2) d 1 d 2 \\
& E_{\text {coul }}^{(3)}=\frac{1}{3} \sum_{I, J} \iiint g_{x c}^{I, J, J}(1,2,3) \delta \rho^{I}(1) \delta \rho^{J}(2) \delta \rho^{J}(3) d 1 d 2 d 3
\end{aligned}
$$

where three center integrals have been neglected in $E_{\text {coul }}^{(3)}$. Semiempirical approximations are applied to this final expression to obtain,

$$
\begin{aligned}
& E_{\text {coul }}^{(2)}(\mathbf{R})=\frac{1}{2} \sum_{I, J} \gamma_{I, J}\left(R_{I, J}\right) \Delta q_{I} \Delta q_{J} \\
& E_{\text {coul }}^{(3)}(\mathbf{R})=\frac{1}{3} \sum_{I, J} \Gamma_{I, J}\left(R_{I, J}\right) \Delta q_{I}^{2} \Delta q_{J}
\end{aligned}
$$


Here, $\Delta q_{I}$ is the change in the Mulliken charge of atom $I$ upon formation of the molecule,

$$
\begin{aligned}
q_{I} & =\sum_{\mu \in I} \sum_{\nu} P_{\mu, \nu} s_{\mu, \nu} \\
\Delta q_{I} & =q_{I}-q_{I}^{0}
\end{aligned}
$$

$\gamma_{I, J}$ is an approximate electron repulsion integral (ERI), and $\Gamma_{I, J}$ is the derivative of $\gamma_{I, J}$ with respect to $\Delta q_{I}\left(\Delta q_{J}\right)$. Minimizing the energy expression (Equation (22)) while maintaining orthonormality of the MOs can be done in the usual way, using the method of Lagrangian parameters. The final orbital Hamiltonian matrix is then,

$$
f_{\mu \in I, \nu \in J}=f_{\mu \in I, \nu \in J}^{\mathrm{DFTB}}+f_{\mu \in I, \nu \in J}^{\mathrm{SCC}},
$$

where the first term on the right-hand side is just the one obtained from the original DFTB method without self-consistent charges. The SCC contribution is given by,

$$
\begin{aligned}
& f_{\mu \in I, \nu \in J}^{\mathrm{SCC}}=f_{\mu \in I, \nu \in J}^{\mathrm{SCC}(2)}+f_{\mu \in I, \nu \in J}^{\mathrm{SCC}(3)} \\
& f_{\mu \in I, \nu \in J}^{\mathrm{SCC}(2)}=\frac{s_{\mu \in I, \nu \in J}}{2} \sum_{K}\left(\gamma_{J, K}+\gamma_{K, I}\right) \Delta q_{K} \\
& f_{\mu \in I, \nu \in J}^{\mathrm{SCC}(3)}=s_{\mu \in I, \nu \in J} \sum_{\tau \in K}\left[\frac{1}{3}\left(\Delta q_{I} \Gamma_{I, K}+\Delta q_{J} \Gamma_{J, K}\right)+\frac{\Delta q_{K}}{6}\left(\Gamma_{K, I}+\Gamma_{K, J}\right)\right]
\end{aligned}
$$

As $\Delta q_{K}$ depends on the MO coefficients $c_{\mu, i}$, then Equation (17) must now be solved self-consistently.

It remains to say a word about how the ERI are approximated. In early work, diagonal elements of $\gamma_{I, J}$ were calculated using Pariser's observation [63] that,

$$
\gamma_{I, I}=\mathrm{IP}_{I}-\mathrm{EA}_{I}
$$

where $\mathrm{IP}_{I}$ and $\mathrm{EA}_{I}$ are respectively the ionization potential and electron affinity of atom $I$. In DFTB3, the $\gamma_{I, I}$ is estimated using Janak's theorem by taking a numerical derivative of the HOMO energy with respect to its occupation number in the neutral atom [62]. The off-diagonal elements are calculated using the zero differential overlap (ZDO) approximation for electron repulsion integrals,

$$
(\mu \nu \| \kappa \lambda) \approx \delta_{\mu, \nu} \delta_{\kappa, \lambda}(\mu \mu \| \kappa \kappa)
$$

and spherically-symmetric $s$ functions on each of the two atoms,

$$
\gamma_{I, J}=\gamma_{s \in I, s \in J}
$$

in order to avoid well-known invariance problems in semiempirical integral evaluation. The exact type of $s$ function used to evaluate the $\gamma_{I, J}$ may vary depending on the implementation of DFTB [53,56]. The parameterization of the DFTB3 $\Gamma$ parameters is explained in [62] along with other details not explained in this brief overview.

We do not expect DFTB to behave like exact DFT. We do not even expect it to be as accurate as density-functional approximations (DFAs). We regard DFTB as a more approximate form of a DFA, which can be applied to answer properly-posed questions for larger or more complex systems than can normally be treated with DFAs. As such, this paper focuses on how well DFTB can capture trends. 


\section{Computational Details}

Three different programs were used in the present work; namely the DFTB+ program [64] for DFTB calculations, GAUSSIAN09 [65] for Hartree-Fock (HF), DFT and OVGF calculations and the post-SIESTA [66] program MBPT_LCAO [19,67] for $G W$ calculations. The DFTB calculations are entirely internally consistent in the sense that DFTB calculations were carried out at geometries optimized at the same level of the DFTB methodology. Vibrational frequencies were calculated to verify true minima by the absence of imaginary vibrational frequencies.

Many of the other calculations are also completely internally consistent in the sense that the same basic method (and basis set) was used for geometry optimizations as for subsequent property calculations. However, some of our calculations are property calculations using one method at a geometry optimized with another basis set. It is convenient to describe these calculations using a common notation for a "theoretical model chemistry": method used/basis set [68]. If different models are used to optimize the geometry than for property calculations at that geometry, then we will use the notation: property model//geometry used. For example, OVGF/6-31G//B3LYP/6-31G** means that the OVGF calculations with the 6-31G basis set were carried out at the geometry optimized using DFT with the B3LYP functional and the 6-31G** basis set.

DFTB + All of the DFTB calculations reported in this paper were performed by using Version 4 Release 1.2 of the DFTB+ program [64] and the parameter set in the 3ob (the third-order DFTB (DFTB3) organic and biochemistry parameter set) Slater-Koster file downloaded from the DFTB site [69]. The self-consistent charges (SCC) were full third-order calculations activated by using the keyword ThirdOrderFull $=$ Yes and specifying HubbardDerivs for all atoms $(\mathrm{C}, \mathrm{H}, \mathrm{N}$ and $\mathrm{S})$. The $3 \mathrm{ob}$ set has been optimized at the DFTB3 level for bio and organic molecules containing carbon, hydrogen, nitrogen and oxygen [70]. It also contains additional parameters for sulfur and phosphorus [71], which, however, were not needed in this study. Clearly, any conclusions of our assessment of DFTB are specific to this parameter set.

We noticed that some molecules with degenerate orbitals would not converge without the use of fractional occupation numbers. For these molecules, convergence was obtained by using a Fermi orbital occupancy distribution with temperatures in the range 10-40 K.

GAUSSIAN09 Several types of calculations were carried out using the GAUSSIAN09 program [65], namely DFT (LDA and B3LYP), HF and OVGF calculations. Two different sets of HF calculations were carried out. The first set (HF/6-31G*//HF/6-31G*) was carried out using the 6-31G(d) (also known as the $6-31 \mathrm{G}^{*}$ ) basis set $[72,73]$. Geometries were re-optimized using the HF method, and this basis set and minima were verified by checking that all vibrational frequencies were real. The second set (HF/6-31G//B3LYP//6-31G**) was carried out as a step in our OVGF calculations. For PCBM, even this was too computationally demanding for OVGF calculations, and we report only results (HF/STO-3G//B3LYP//6-31G**) with the minimal STO-3G basis set [74]. 
DFT calculations with GAUSSIAN09 used either the LDA or the B3LYP functional. LDA calculations used the SVWN (Slater-Vosko-Wilk-Nusair, a Gaussian09 keyword) option, which corresponds to the usual Vosko, Wilk and Nusair parameterization of the random phase approximation (RPA) results for the homogeneous electron gas [75]. The B3LYP [76] functional is a variation on the B3P functional [77]. Both the LDA and B3LYP calculations used the 6-31G(d) (also known as the 6-31G*) basis set $[72,73]$. Furthermore, in both cases, the minima from geometry optimizations were verified, and no imaginary frequencies were found. Thus, the reported results are at the LDA/6-31G*//LDA/6-31G* and B3LYP/6-31G*//B3LYP/6-31G* levels of theoretical model chemistry. In addition, B3LYP geometry optimizations were carried out with the larger 6-31G(d,p) (also known as 6-31G**) basis set [72,73], and these B3LYP/6-31G** optimized geometries were used for all of our Green's function calculations.

Our outer-valence Green's function (OVGF) calculations were carried out using the 6-31G [72] basis set at the B3LYP/6-31G** optimized geometries. The OVGF calculations proved to be computationally demanding, and we were not able to use a more complete basis sets for these molecules. For the PCBM molecule, we were obliged to use the even smaller STO-3G basis set [74] (OVGF/STO-3G//B3LYP/6-31G**). Convergence with respect to basis set completeness was checked in the case of pentacene by also carrying out calculations using the larger $6-311++\mathrm{G}^{* *}$ basis set $[78,79]$. In going from the $6-31 \mathrm{G}$ to the $6-311++\mathrm{G}^{* *}$ basis set at the B3LYP/6-31G** optimized geometry, the OVGF HOMO energy of pentacene changed by $0.49 \mathrm{eV}$, while the corresponding HF HOMO energy changed by only about $0.18 \mathrm{eV}$. This is consistent with the usual observation that MBPT (many-body perturbation theory) calculations require larger basis sets than HF in order to properly describe in-out and angular correlation effects. For this reason, our OVGF calculations should be considered as indicative, but certainly not conclusive, as we do not believe they are well converged with respect to the orbital basis set used.

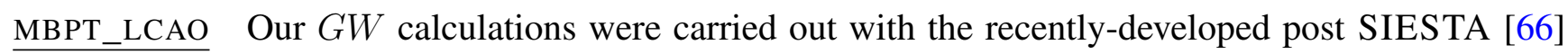
MBPT_LCAO code $[19,67]$ at the B3LYP/6-31G** optimized geometries previously optimized with GAUSSIAN09. The SIESTA part of the calculation used the norm-conserving Trouillier-Martins-type pseudopotential together with a double-zeta polarized numerical atomic orbital basis set. Computational parameters were chosen in a uniform manner to be the same for all molecules, in such a way that calculations for the most computationally-demanding molecule (PCBM) still fit into the random-access memory (RAM). Even so, our PCBM calculation required nine days and $8 \mathrm{~h}$ on an Intel 12 core/190-GB RAM machine (model name: Intel(R) Xeon(R) 2.40-GHz E5645 CPU). The spatial extension of the orbitals was determined by the parameter EnergyShift for which we could afford the value EnergyShift $=50 \mathrm{meV}$ with a frequency resolution of $0.12 \mathrm{eV}$. The calculations are $G_{0} W_{0}$ calculations constructed in the following way: LDA SIESTA orbitals were used as an initial guess to carry out a self-consistent HF calculation, producing HF orbitals and orbital energies. These are then used in a one-shot $G W$ calculation that might best be termed a $G_{0} W_{0}(\mathrm{HF})$ calculation. The IPs and EAs were estimated in two ways. 
In the first, the density-of-states (DOS) was calculated using the expression,

$$
\operatorname{DOS}(\omega)=(\theta(\omega-\mu)-\theta(\mu-\omega)) \frac{1}{\pi} \Im \sum_{s}\left\langle\psi_{s}|\hat{G}(\omega)| \psi_{s}\right\rangle
$$

on an energy grid, the extent of which is governed by the maximal difference of the DFT orbital energies multiplied with a factor 1.15 . The quantity $\mu$ is the "Fermi energy" separating IP solutions from EA solutions. Let us see the relation to the IPs and EAs. Since,

$$
G(1,2 ; \omega)=\sum_{I} \frac{\psi_{I}(1) \psi_{I}^{*}(2)}{\omega-\epsilon_{I}-i \eta}+\sum_{A} \frac{\psi_{A}(1) \psi_{A}^{*}(2)}{\omega-\epsilon_{A}+i \eta}
$$

in terms of ionization $(I)$ and electron attachment $(A)$ Dyson orbitals $\psi_{S}$ and energies $\epsilon_{S}$. Here, $\eta$ is infinitesimal in principle, but is a small real number in practical calculations. Then, using the completeness of the MO basis set $\left(\psi_{s}\right)$, Equation (33) reduces to,

$$
\operatorname{DOS}(\omega)=\sum_{I} S_{I} L\left(\omega ; \epsilon_{I}, \eta\right)+\sum_{A} S_{A} L\left(\omega ; \epsilon_{A}, \eta\right)
$$

where,

$$
L\left(\omega ; \epsilon_{S}, \eta\right)=\frac{1}{\pi} \frac{\eta}{\left(\omega-\epsilon_{S}\right)^{2}+\eta^{2}}
$$

is the usual Lorentzian function and $S_{S}$ is the pole strength of the transition. We thus expect the DOS to have maxima at the IPs and EAs. The scale parameter $\eta$ is the half-width at half-maximum of the Lorentzian. This scale parameter was chosen to be $0.24 \mathrm{eV}$ in our calculations as justified elsewhere [80]. Thus, our DOS IP is obtained by fitting the highest peak below the gap in the DOS to a third-order polynomial and calculating the maximum of the polynomial. The EA is determined in a similar way, by fitting the lowest peak above the gap.

The second way of estimating IPs and EAs is referred to in this paper as the quasiparticle (QP) method. It assumes that the starting MOs, $\psi_{s}$, are a good approximation to the final Dyson amplitudes, which is usually the case for solutions with significant pole strength (i.e., when the self-energy varies sufficiently slowly as is typically the case close to the HOMO-LUMO gap). Then,

$$
\omega_{s}^{(\mathrm{QP})}=\left\langle\psi_{s}\left|\hat{h}_{H}\right| \psi_{s}\right\rangle+\Re\left\langle\psi_{s}\left|\Sigma_{x c}^{G_{0} W_{0}}\left(\omega^{(\mathrm{QP})}\right)\right| \psi_{s}\right\rangle
$$

is solved graphically (Figure 2). The advantage of using the QP method over using the DOS method is that the peaks may be more fully resolved and assigned to a corresponding MO, even for higher poles. 


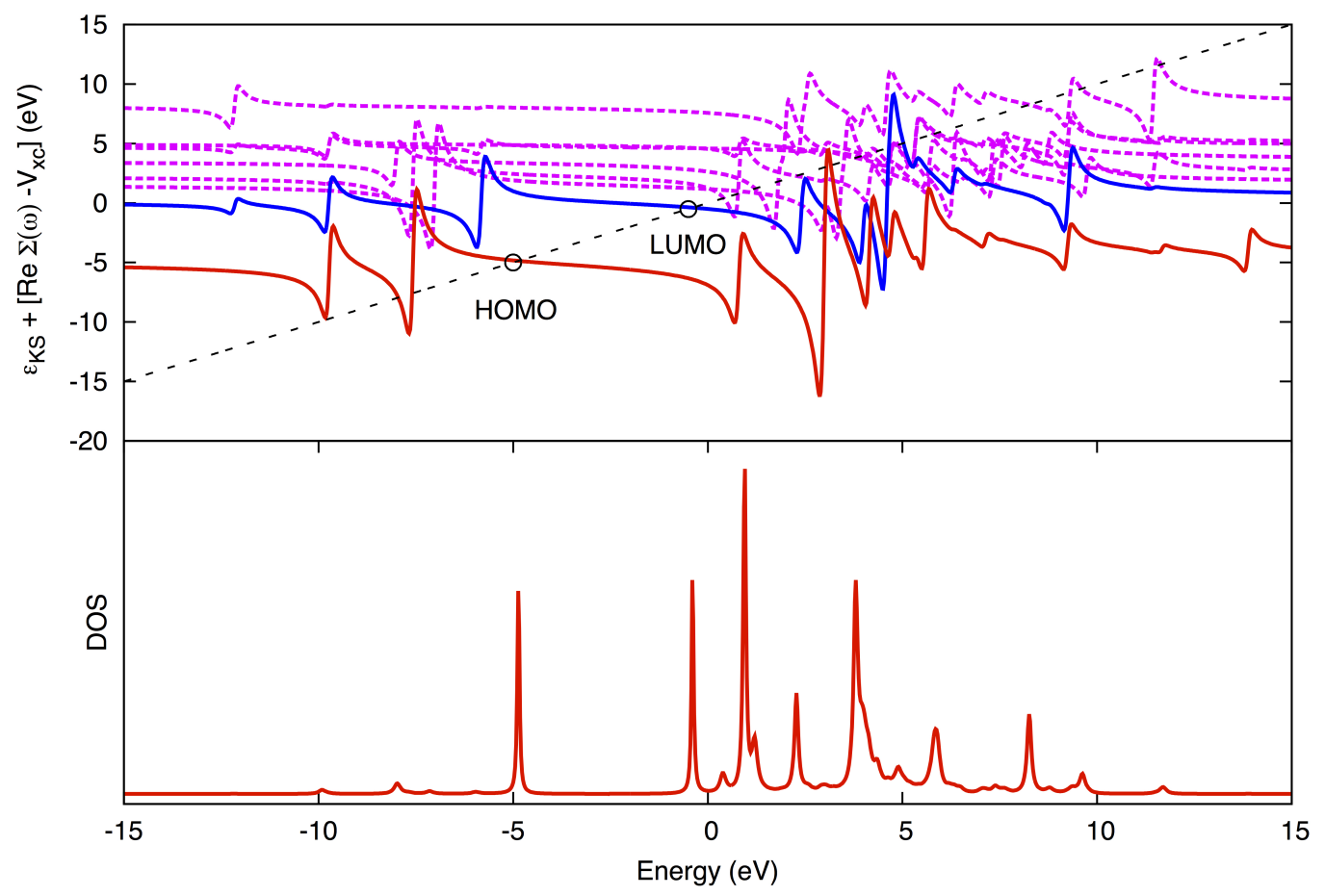

Figure 2. Graphical solution in the quasiparticle (QP) method. (Top) The diagonal line corresponds to $\omega$, while the horizontal curves correspond to $\left\langle\psi_{s}\left|\hat{h}_{H}\right| \psi_{s}\right\rangle+$ $\Re\left\langle\psi_{s}\left|\Sigma_{x c}^{G_{0} W_{0}}(\omega)\right| \psi_{s}\right\rangle$ for different states, HOMO being red, LUMO blue and higher states jagged magenta. The QP solutions for HOMO and LUMO are marked with circles. (Bottom) The corresponding density-of-states (DOS). Pole strengths (the magnitude of the peaks in the DOS) are obtained from the slope where the diagonal line crosses the horizontal curves.

\section{Small Molecules}

In this section and in the next section, we will explain our protocol for assessing DFTB for calculating IPs and EAs and give the results of our assessment. A division has been made between small molecules (this section) and medium-sized molecules (next section). This is because much more information is available for benchmarking small molecules while reliable information for benchmarking medium-sized molecules of interest for organic solar cell applications is much more scarce. In this section, we first review our protocol and illustrate how it works for known results from the literature. We then go on to apply our protocol to the assessment of DFTB calculations.

Benchmark Datasets The identification and tabulation of reliable comparison data are key parts of the test cycle part of the development and successful implementation of a theoretical model for practical applications. The very important and, indeed essential, work involved in establishing such "benchmark datasets" (BDSs) can be substantial and relatively thankless, as it may be regarded primarily as "literature work", rather than as "new science." An especially well-known example of such a test set is Charlette E. Moore's tables of atomic spectra, now freely available from the American National Institute of Standards and Technology (NIST, [81]), which has proven essential in testing different approaches to quantum chemistry. 
For small molecules, experimental and quantum chemistry ionization potentials (IPs) are readily available from the NIST Computational Chemistry Comparison and Benchmark Database (CCCBDB, [82]). As we have chosen to use the same BDS as in previous work by Hamel et al. [40] for the molecules shown in Figure 3, we have simply taken their values for our small-molecule BDS. This gives a total of 56 IPs (Tables 1 and 2). Note that these small molecules cannot be used to benchmark electron affinities (EAs) in the sense that most do not bind an extra electron. Hence, we will not assess the quality of DFTB EAs for these small molecules.

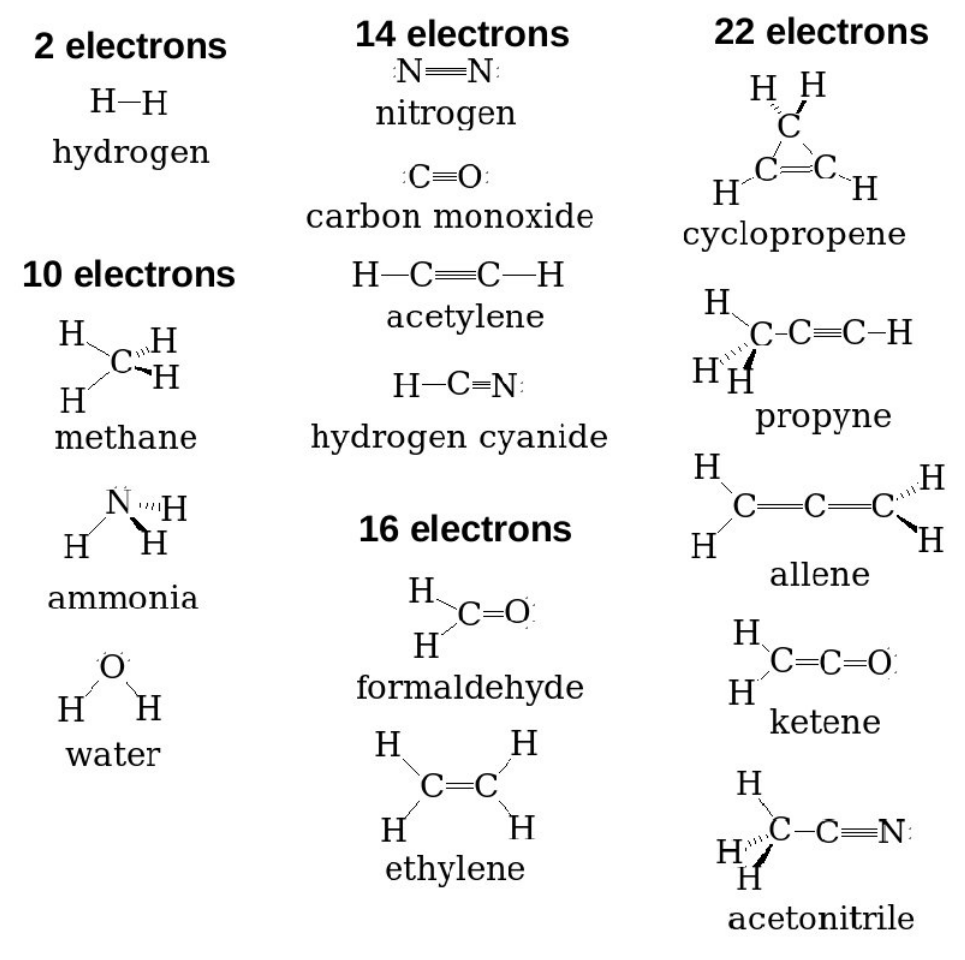

Figure 3. The 15 small molecules treated here.

Analytical Protocol Figure 4 simply takes the data from Hamel et al. [40] to make a correlation plot of minus LDA orbital energies (LDA “Koopmans' theorem IPs") versus experimental IPs. It is clear that the LDA Koopmans' theorem IPs underestimate the experimental IPs, but that also there is an excellent linear correlation between the LDA Koopmans' theorem IPs and the experimental IPs for these small molecules. We are thus faced with a dilemma regarding how good LDA Koopmans' IPs are as a model for experimental IPs, and the same dilemma may arise when trying to assess other methods. At this point, we may adapt one of two different quantum chemical philosophies, which Coulson has famously called "Group I" and "Group II" [83] (see also [84,85]). Roughly speaking, Group I focuses on getting good numbers for individual molecules, so that the best theory would have a slope $m$ near unity and an intercept $b$ near zero, while Group II focuses on the ability to predict trends, so that the best theory is one where there is a systematic and, hence, predictive correlation between theory and experiment, even if the two give quantitatively different results. 
Table 1. DFTB+ ionization potentials (IPs) (eV) for small molecules. SCC, self-consistent charge.

\begin{tabular}{|c|c|c|c|c|c|c|}
\hline \multirow[t]{2}{*}{ Molecule } & \multirow[t]{2}{*}{ Orbital } & \multirow[t]{2}{*}{ Experiment ${ }^{a}$} & \multicolumn{2}{|c|}{ Without SCC } & \multicolumn{2}{|c|}{ With SCC } \\
\hline & & & $-\epsilon_{i}$ & $\Delta \mathrm{SCF}$ & $-\epsilon_{i}$ & $\Delta \mathrm{SCF}$ \\
\hline \multicolumn{7}{|c|}{2 electrons } \\
\hline hydrogen $\left(\mathrm{H}_{2}\right)$ & $1 a_{g}$ & 15.43 & 9.26 & 9.26 & 9.26 & 14.67 \\
\hline \multicolumn{7}{|c|}{10 electrons } \\
\hline methane $\left(\mathrm{CH}_{4}\right)$ & $1 t_{2}$ & 14.3 & 9.17 & 9.17 & 9.00 & 13.41 \\
\hline $\operatorname{ammonia}\left(\mathrm{NH}_{3}\right)$ & $3 a_{1}$ & 10.7 & 7.73 & 7.73 & 6.95 & 12.18 \\
\hline \multirow[t]{4}{*}{ water $\left(\mathrm{H}_{2} \mathrm{O}\right)$} & $1 b_{1}$ & 12.62 & 9.04 & 9.04 & 7.64 & 13.31 \\
\hline & $3 a_{1}$ & 14.74 & 10.28 & - & 9.03 & - \\
\hline & $1 b_{2}$ & 18.51 & 12.31 & - & 11.54 & - \\
\hline & $2 a_{1}$ & 32.2 & 24.65 & - & 23.48 & - \\
\hline \multicolumn{7}{|c|}{14 electrons } \\
\hline \multirow{3}{*}{ nitrogen $\left(\mathrm{N}_{2}\right)$} & $3 \sigma_{g}$ & 15.6 & 9.70 & 9.70 & 9.70 & 15.33 \\
\hline & $1 \pi$ & 16.98 & 11.16 & - & 11.16 & - \\
\hline & $2 \sigma_{u}$ & 18.78 & 13.98 & - & 13.98 & - \\
\hline \multirow[t]{3}{*}{ carbon monoxide $(\mathrm{CO})$} & $5 \sigma$ & 14.01 & 9.16 & 9.16 & 9.16 & 14.47 \\
\hline & $1 \pi$ & 16.91 & 11.21 & - & 11.18 & - \\
\hline & $4 \sigma$ & 19.72 & 13.67 & - & 13.64 & - \\
\hline \multirow[t]{4}{*}{ acetylene $\left(\mathrm{C}_{2} \mathrm{H}_{2}\right)$} & $1 \pi_{u}$ & 11.49 & 8.33 & 8.33 & 7.86 & 12.23 \\
\hline & $3 \sigma_{g}$ & 16.7 & 10.29 & - & 10.11 & - \\
\hline & $2 \sigma_{u}$ & 18.7 & 12.66 & - & 12.45 & - \\
\hline & $2 \sigma_{g}$ & 23.5 & 17.87 & - & 17.44 & - \\
\hline \multirow[t]{3}{*}{ hydrogen cyanide (HCN) } & $1 \pi$ & 13.8 & 9.79 & 9.79 & 9.30 & 14.12 \\
\hline & $5 \sigma$ & 14.15 & 9.84 & - & 9.31 & - \\
\hline & $4 \sigma$ & 19.68 & 12.42 & - & 13.11 & - \\
\hline \multicolumn{7}{|c|}{16 electrons } \\
\hline \multirow[t]{5}{*}{ formaldehyde $\left(\mathrm{CH}_{2} \mathrm{O}\right)$} & $2 b_{2}$ & 10.9 & 7.06 & 7.06 & 6.43 & 10.98 \\
\hline & $1 b_{1}$ & 14.5 & 10.47 & - & 9.92 & - \\
\hline & $5 a_{1}$ & 16.1 & 10.78 & - & 10.37 & - \\
\hline & $1 b_{2}$ & 17. & 11.08 & - & 10.96 & - \\
\hline & $4 a_{1}$ & 21.4 & 14.56 & - & 14.43 & - \\
\hline \multirow[t]{6}{*}{ ethylene $\left(\mathrm{C}_{2} \mathrm{H}_{4}\right)$} & $1 b_{2 u}$ & 10.68 & 7.75 & 7.75 & 7.36 & 10.94 \\
\hline & $1 b_{2 g}$ & 12.79 & 8.10 & - & 7.88 & - \\
\hline & $3 a_{g}$ & 14.8 & 9.65 & - & 9.35 & - \\
\hline & $1 b_{3 u}$ & 15.18 & 10.37 & - & 10.09 & - \\
\hline & $2 b_{1 u}$ & 19.1 & 13.20 & - & 12.91 & - \\
\hline & $2 a_{g}$ & 23.59 & 17.60 & - & 17.24 & - \\
\hline
\end{tabular}

${ }^{a}$ Taken from [40]. 
Table 2. DFTB+ IPs (eV) for small molecules (continued).

\begin{tabular}{|c|c|c|c|c|c|c|}
\hline \multirow[t]{2}{*}{ Molecule } & \multirow[t]{2}{*}{ Orbital } & \multirow[t]{2}{*}{ Experiment $^{a}$} & \multicolumn{2}{|c|}{ Without SCC } & \multicolumn{2}{|c|}{ With SCC } \\
\hline & & & $-\epsilon_{i}$ & $\triangle \mathrm{SCF}$ & $-\epsilon_{i}$ & $\Delta \mathrm{SCF}$ \\
\hline \multicolumn{7}{|c|}{22 electrons } \\
\hline \multirow[t]{7}{*}{ cyclopropene $\left(\mathrm{C}_{3} \mathrm{H}_{4}\right)$} & $2 b_{2}$ & 9.86 & 6.75 & 6.75 & 6.28 & 10.00 \\
\hline & $2 b_{1}$ & 10.89 & 7.64 & - & 7.20 & - \\
\hline & $6 a_{2}$ & 12.7 & 8.59 & - & 8.11 & - \\
\hline & $1 b_{2}$ & 15.09 & 10.01 & - & 9.52 & - \\
\hline & $5 a_{1}$ & 16.68 & 10.36 & - & 10.04 & - \\
\hline & $2 b_{1}$ & 18.3 & 13.01 & - & 12.69 & - \\
\hline & $4 a_{1}$ & 19.6 & 13.95 & - & 13.48 & - \\
\hline \multirow[t]{4}{*}{ propyne $\left(\mathrm{CH}_{3} \mathrm{CCH}\right)$} & $2 e$ & 10.37 & 7.71 & 7.71 & 7.18 & 10.87 \\
\hline & $1 e$ & 14.4 & 9.71 & - & 9.65 & - \\
\hline & $7 a_{1}$ & 15.5 & 10.00 & - & 9.69 & - \\
\hline & $6 a_{1}$ & 17.2 & 11.91 & - & 11.56 & - \\
\hline \multirow[t]{4}{*}{ allene $\left(\mathrm{C}_{3} \mathrm{H}_{4}\right)$} & $2 e$ & 10.02 & 7.21 & 7.21 & 6.85 & 10.43 \\
\hline & $1 e$ & 14.75 & 9.95 & - & 9.67 & - \\
\hline & $3 b_{2}$ & 14.75 & 9.98 & - & 9.68 & - \\
\hline & $4 a_{1}$ & 17.3 & 11.93 & - & 11.66 & - \\
\hline \multirow[t]{6}{*}{ ketene $\left(\mathrm{CH}_{2} \mathrm{CO}\right)$} & $2 b_{1}$ & 9.8 & 6.27 & 6.27 & 6.20 & 10.21 \\
\hline & $2 b_{2}$ & 14.2 & 9.24 & - & 9.23 & - \\
\hline & $1 b_{1}$ & 15. & 10.62 & - & 10.71 & - \\
\hline & $1 b_{2}$ & 16.3 & 10.93 & - & 10.97 & - \\
\hline & $7 a_{1}$ & 16.8 & 11.07 & - & 11.14 & - \\
\hline & $6 a_{1}$ & 18.2 & 12.94 & - & 12.86 & - \\
\hline \multirow[t]{4}{*}{ acetonitrile $\left(\mathrm{CH}_{3} \mathrm{CN}\right)$} & $2 e$ & 12.08 & 8.66 & 8.66 & 8.45 & 12.30 \\
\hline & $7 a_{1}$ & 13.11 & 9.69 & - & 8.96 & - \\
\hline & $1 e$ & 15.5 & 10.25 & - & 10.36 & - \\
\hline & $6 a_{1}$ & 17.4 & 11.81 & - & 11.77 & - \\
\hline
\end{tabular}

${ }^{a}$ Taken from [40].

In the spirit of Group I, we should keep in mind that even the best computed molecular vertical IPs have an average error of about $0.2 \mathrm{eV}$ compared to experimental vertical IPs determined by peak maxima in photoelectron spectra, because of the neglect of vibrational (i.e., Franck-Condon) effects. In the spirit of Group II, we have taken the standard error,

$$
\Delta y=\sqrt{\frac{\mathcal{E}_{y}}{N-2}}
$$

where,

$$
\mathcal{E}_{y}=\sum_{i=1, N}\left(y_{i}-m x_{i}-b\right)^{2}
$$


as the most transferable estimate of the goodness of the fit. However, if we now take the fit values of $m$ and $b$ found above for a particular method and then use them in the formula,

$$
x=\frac{1}{m} y-\frac{b}{m}
$$

to try to estimate the error in the predicted value of BDS values corresponding to the results of the model, then the relevant standard error is:

$$
\Delta x=\sqrt{\frac{\mathcal{E}_{x}}{N-2}}=\frac{\Delta y}{|m|},
$$

where:

$$
\mathcal{E}_{x}=\sum_{i=1, N}\left(x_{i}-\frac{1}{m} y_{i}+\frac{b}{m}\right)^{2}=\frac{\mathcal{E}_{y}}{m^{2}} .
$$

Thus, $\Delta x$ is the relevant measure of the predictability of a given model.

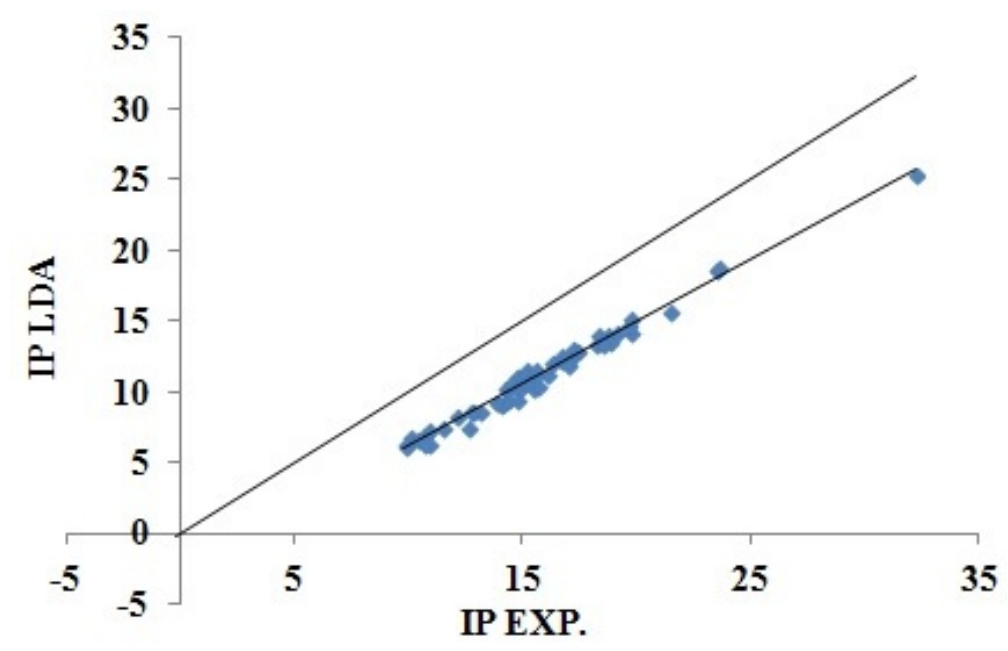

(a)

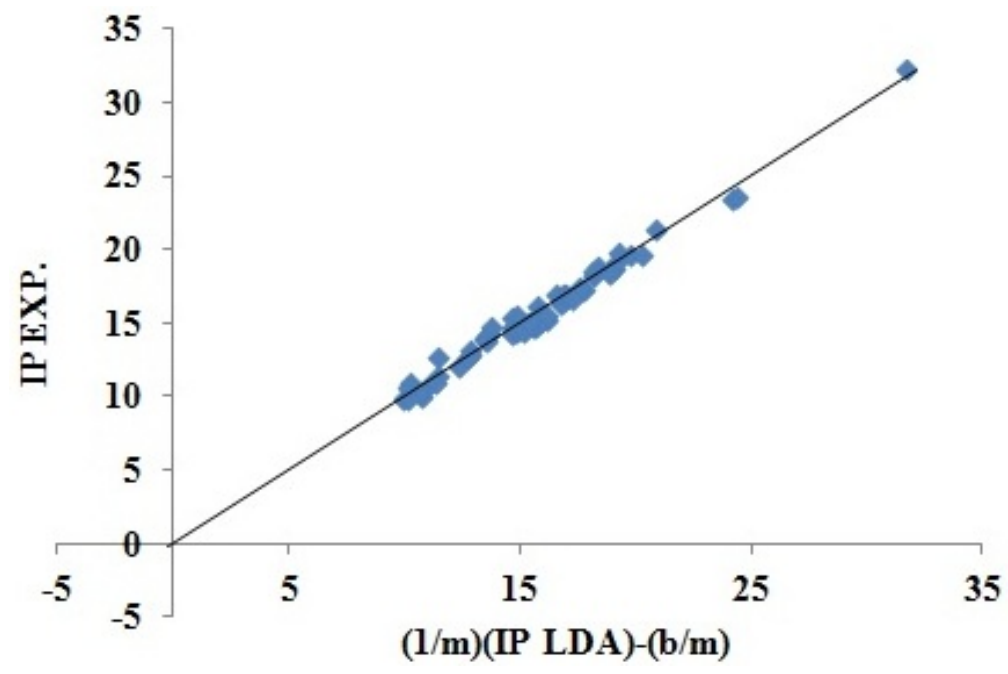

(b)

Figure 4. (a) Correlation plot for LDA “Koopmans' theorem IPs" versus experimental IPs [40]; (b) inversion plot for the same data constructed using Equation (40) to show the "predictive ability" of the LDA least squares fit. The units for both axes in these plots are eV. 
The results of a statistical analysis of the results in [40] for least square fits of various theoretical methods to experiments are shown in Tables 3 and 4. Consistent with Figure 4, Table 3 shows that the DFT LDA Koopmans' theorem (KT) underestimates by $4-6 \mathrm{eV}$ (i.e., $y-x=(m-1) x+b$ is $-3.87 \mathrm{eV}$ at $x=10 \mathrm{eV}$ and $-6.23 \mathrm{eV}$ at $x=30 \mathrm{eV}$ ) and, so, is a dismal failure from the point of view of Coulson Group I. This has been explained in terms of the particle number discontinuity in the DFT exchange-correlation (xc) potential [86]. The Hartree-Fock (HF) KT IPs are better from the point of view of Coulson Group I, but tend to slightly overestimate the experimental IPs. This is consistent with early literature, which proposed as a rule of thumb that agreement between HF KT and photoelectron spectroscopy (PES) IPs could be improved by multiplying the HF KT values by a factor of less than one (e.g., 0.92 or the "8\% rule" [87,88]). Surprisingly, localizing the HF exchange operator by the optimized effective, potential method (OEP) so as to obtain an exact exchange-only xc-potential which includes a derivative discontinuity, leads to much improved absolute agreement between KT and experimental IPs with a slightly reduced standard error $(\Delta y)$. Part of the reason for the smaller value of $\Delta y$ is that the OEP corrects misorderings in the KT IPs, which are present when KT is used with the nonlocal HF exchange operator. Note that the OEP procedure makes use of the constraint that the highest occupied molecular orbital (HOMO) energy be identical for HF and OEP when both are evaluated with the OEP orbitals [41]. Thus, the OEP HOMO energy is almost, but not exactly, the same as the HF HOMO energy.

Table 3. Fitting data for outer valence IPs: $y=m x+b$, where $x$ is the experimental IP and $y$ is the calculated IP, both in eV. HF, Hartree-Fock; OEP, optimized effective potential; LDA, local density approximation; DFTB, density-functional tight binding (without self-consistent charge); SCC-DFTB, self-consistent charge DFTB.

\begin{tabular}{|c|c|c|c|c|}
\hline Method & $m$ (Unitless) & $b(\mathrm{eV})$ & $\Delta y(\mathrm{eV})$ & $\Delta x(\mathbf{e V})$ \\
\hline \multicolumn{5}{|c|}{ Koopmans' Theorem } \\
\hline $\mathrm{OEP}^{a}$ & 0.958 & 0.748 & 0.659 & 0.688 \\
\hline $\mathrm{HF}^{a}$ & 1.236 & -2.192 & 0.686 & 0.555 \\
\hline $\mathrm{LDA}^{a}$ & 0.882 & -2.691 & 0.474 & 0.537 \\
\hline DFTB $^{b}$ & 0.754 & -1.071 & 0.651 & 0.863 \\
\hline SCC-DFTB $^{b}$ & 0.758 & -1.442 & 0.535 & 0.706 \\
\hline \multicolumn{5}{|c|}{$\triangle \mathrm{SCF}$} \\
\hline $\operatorname{LDA}^{c}$ & 1.65 & 2.54 & 1.65 & 1.85 \\
\hline
\end{tabular}

${ }^{a}$ Calculated from data in $[40] ;{ }^{b}$ present work; ${ }^{c}$ approximate $\triangle \mathrm{SCF}$ values calculated using Slater's transition orbital method. "rTS" (restricted transition state) values from [89]. 
Table 4. Fitting data for first IPs [40]: $y=m x+b$, where $x$ is the experimental IP and $y$ is the theoretical IP, both in eV. HF, Hartree-Fock; OEP, optimized effective potential; LDA, local density approximation; DFTB, density-functional tight binding (without self-consistent charge); SCC-DFTB, self-consistent charge DFTB.

\begin{tabular}{|c|c|c|c|c|}
\hline Method & $m$ (Unitless) & $b(\mathrm{eV})$ & $\Delta y(\mathrm{eV})$ & $\Delta x(\mathrm{eV})$ \\
\hline \multicolumn{5}{|c|}{ Koopmans' Theorem } \\
\hline $\mathrm{HF}^{a}$ & 1.143 & -1.270 & 0.617 & 0.540 \\
\hline $\mathrm{OEP}^{a}$ & 1.145 & -1.294 & 0.613 & 0.535 \\
\hline $\operatorname{LDA}^{a}$ & 0.727 & -1.064 & 0.374 & 0.514 \\
\hline DFTB $^{b}$ & 0.497 & 2.223 & 0.498 & 1.002 \\
\hline SCC-DFTB $^{b}$ & 0.560 & 1.064 & 0.477 & 0.852 \\
\hline \multicolumn{5}{|c|}{$\triangle \mathrm{SCF}$} \\
\hline $\mathrm{LDA}^{c}$ & 1.00 & 0.49 & 0.43 & 0.43 \\
\hline DFTB $^{b}$ & 0.497 & 2.223 & 0.498 & 1.002 \\
\hline SCC-DFTB $^{b}$ & 0.838 & 2.239 & 0.534 & 0.649 \\
\hline
\end{tabular}

${ }^{a}$ Calculated from data in $[40] ;{ }^{b}$ present work. ${ }^{c}$ approximate $\triangle \mathrm{SCF}$ values calculated using Slater's transition orbital method. "rTS" values from [89].

The outlook changes dramatically from the point of view of Coulson Group II. Looking at $\Delta x$ in Tables 3 and 4, we see that the LDA KT provides as much predictability (indeed somewhat more) as either the HF or OEP KT, indicating that, although shifted, the LDA KT IPs provide an excellent reflection of the trend in the experimental IPs. Closer analysis of why the LDA results are a bit better than the HF or OEP (smaller $\Delta x$ ) shows that the LDA KT IPs have fewer order reversals compared to experimental IPs than do the HF KT IPs. This also occurs when the HF exchange potential is localized in the OEP procedure, though $\Delta x$ is larger for the OEP calculations than for the HF calculations, indicating decreasing predictability upon localization. The above trends do not seem to be well known to most users of quantum chemistry programs, but this does not seem to be the place to spend time and space giving further details (see [41] for an additional discussion). Suffice it to say that the above information is "well-known to those who know about such things" and that further information is contained in the cited papers. It should also be evident that global hybrid functionals containing a nonzero amount of HF exchange will have a behavior in between the LDA and HF, as can be easily checked using information from the NIST CCCBDB, but we shall not pursue this topic further here.

Assessment of DFTB The results of our DFTB + calculations of IPs and EAs are shown in Tables 1 and 2. The results of a statistical analysis of our DFTB results relative to experiments are also shown in Tables 3 and 4.

Since DFTB has been designed as an approximation on DFT, we expect DFTB to behave in much the same way, albeit not exactly, as DFT. We also expect the SCC option to give results closer to DFT than results without the SCC. Let us verify that this is true by first looking at calculations without the SCC option. A trivial consequence of the basic theory (previous section) is that the $\triangle \mathrm{SCF}$ and Koopmans' 
theorem IPs are identical for the original DFTB method, and our results confirm this. Figure 5 shows how DFTB Koopmans' theorem IPs correlate with experiment. As we would hope, this figure is remarkably similar to the corresponding LDA figure (Figure 4). The DFTB Koopmans' theorem IPs are underestimates of the experimental IPs in much the same way as the LDA underestimates Koopmans' theorem. This is consistent with DFTB having been constructed to behave like DFT; a conclusion that is confirmed by Figure 6 showing the excellent correlation between DFTB and LDA Koopmans' theorem IPs. In spite of the similarity of the graphics, the LDA and DFTB values of $\Delta x$ in Table 3 are a reminder that DFTB is significantly less predictive (error bar of $\pm 0.863 \mathrm{eV}$ ) than is the LDA (error bar of $\pm 0.537 \mathrm{eV}$ ).

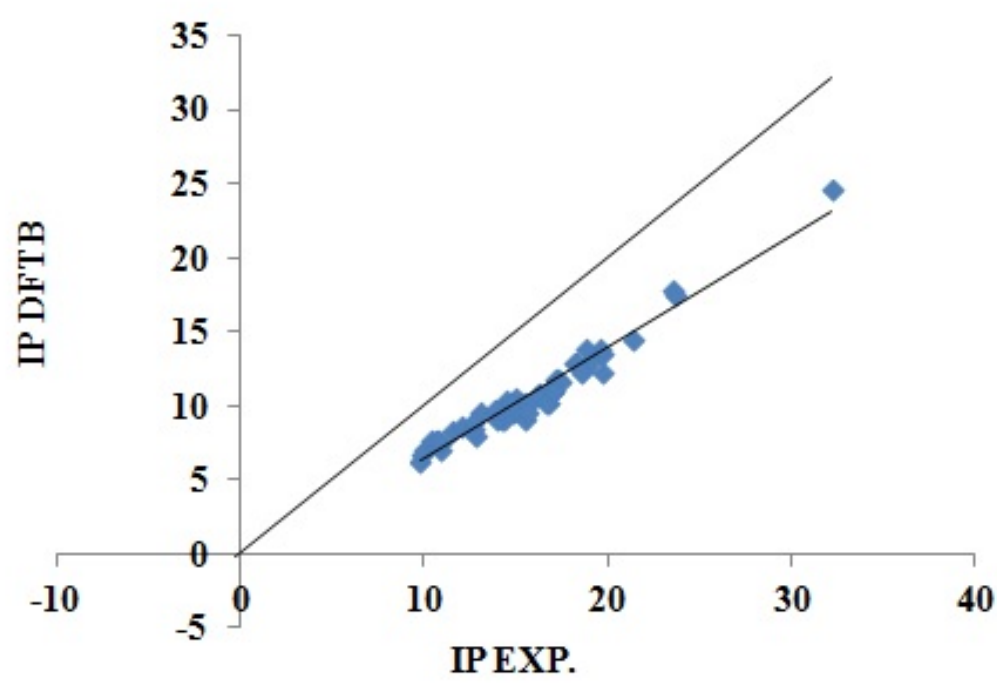

(a)

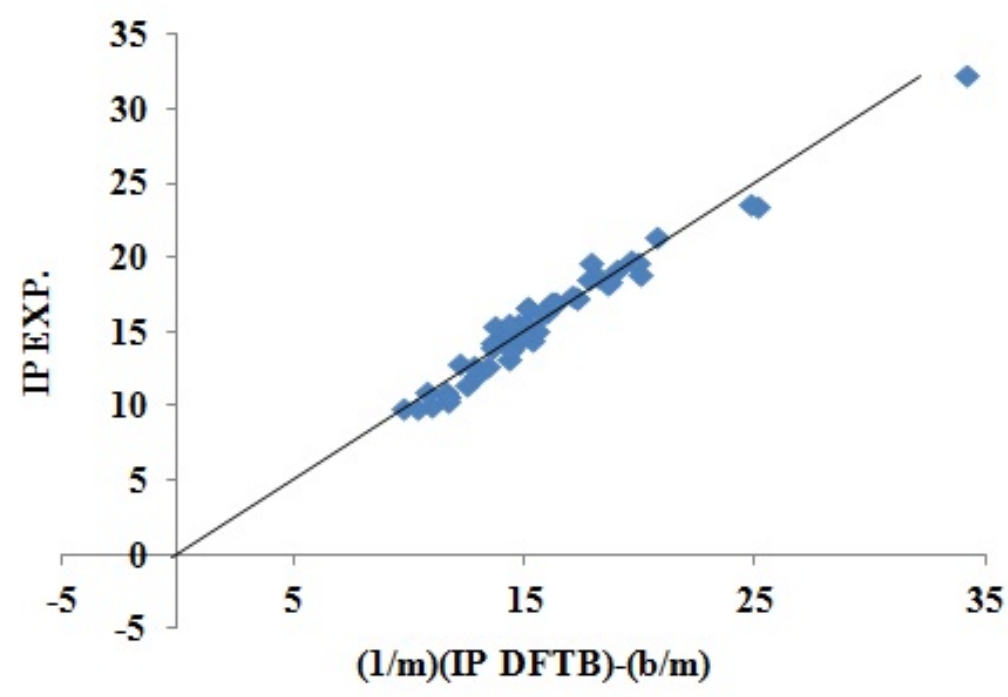

(b)

Figure 5. Correlation plot for DFTB “Koopmans' theorem IPs" versus experimental IPs. (a) Correlation plot for DFTB “Koopmans' theorem IPs" versus experimental IPs; (b) inversion plot for the same data constructed using Equation (40) to show the "predictive ability" of the DFTB least squares fit. The units for both axes in these plots are in eV. 


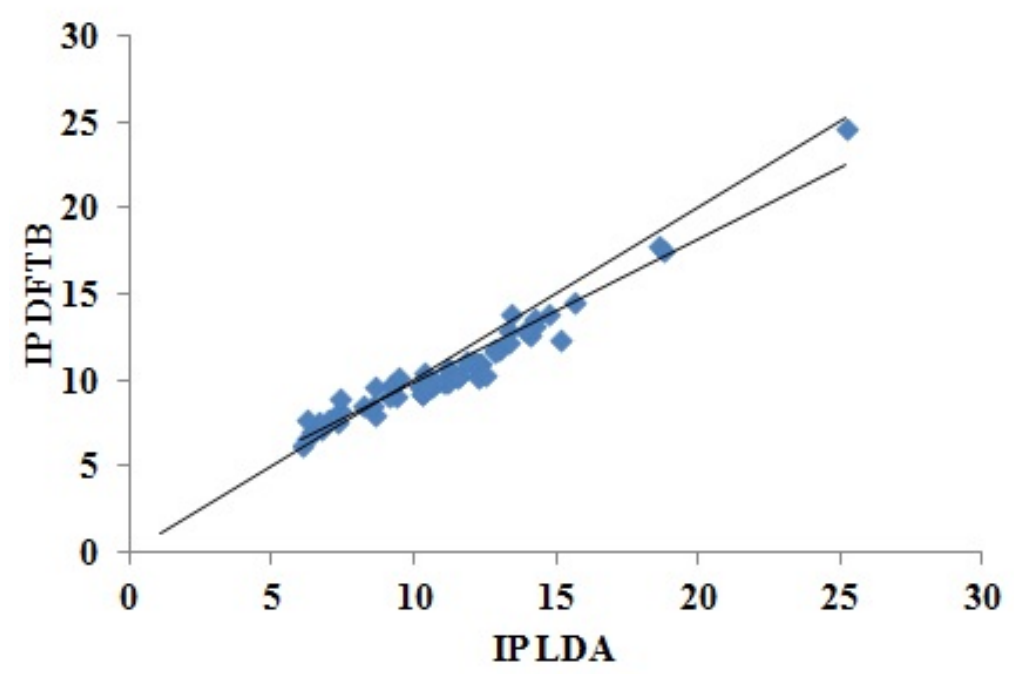

Figure 6. Correlation plot for DFTB versus LDA "Koopmans' theorem IPs." The units for both axes are $\mathrm{eV}$.

According to Tables 3 and 4, including SCC in DFTB leads to a significant partial improvement. While SCC-DFTB still behaves much like the LDA, SCC-DFTB Koopmans' theorem IPs are more predictive (error bar of $\pm 0.706 \mathrm{eV}$ ) than DFTB Koopmans' theorem IPs, though still less than LDA Koopmans' theorem IPs. If we restrict attention to the HOMO, than we can also compare Koopmans' theorem to $\triangle$ SCF SCC-DFTB (which we recall are the same in the case without the SCC). Here, we see that the $\triangle \mathrm{SCF}$ method provides more predictive ability than does the Koopmans' theorem approach. In fact, the SCC-DFTB $\triangle \mathrm{SCF}$ error bar $( \pm 0.649 \mathrm{eV})$ is becoming encouragingly close to the LDA Koopmans' theorem error bar $( \pm 0.514 \mathrm{eV})$, although it is still not as small as that of the "rTS" estimate of the LDA $\triangle$ SCF IPs $( \pm 0.43 \mathrm{eV})$ whose calculation were reported in [89].

\section{Medium-Sized Molecules}

The small molecules in the previous section provide a good idea of how various methods work for calculating IPs for such systems. In particular, we confirmed DFTB much like DFT and placed error bars on the predictive power of the method. Thus, for small molecules, DFT (or some other first-principles method) is preferable to DFTB. However, our interest is in organic electronics, where interesting molecules, and their assembly into realistic systems, rapidly leads to calculations, which are difficult to attack any other way, except using a semi-empirical method, such as DFTB. It is important to see to what extent that what we have learned for small molecules also applies to larger molecules of more interest for organic electronics. This is especially true, since methods tested and found to work well for small molecules do not always perform equally well for large molecules [26]. In this section, we focus on assessing DFTB for the calculation of IPs and EAs for a BDS of "medium-sized" molecules often used in organic electronics (Figure 1).

Choice of Comparison Data Of course, the notion of "small," "medium" and "large" is not well-defined. In the present context, molecules are "medium sized" in the sense that they are the smallest 
molecules of interest for organic electronics, and yet, they are already too large for the NIST CCBDB, which is limited to species with no more than 26 atoms total. Our medium-sized molecules are also large enough that it is experimentally more natural to treat them either in the bulk phase, as either a thin film or in solution electrochemistry, than in the gas phase, though gas phase molecular data are available for a number of the molecules. IPs and EAs for these molecules are frequently determined by thin-film photoelectron or photoemission spectroscopy or by cyclic voltammetry and involve a certain number of assumptions whose accuracy is not always easy to assess. In particular, in thin film photoelectron and photoemission spectroscopy, the difference between the ionization energy of the molecule and the bulk is sometimes referred to as "the polarization energy due to the molecular ion left in the solid after a photoelectron is removed" [90], which is a quantity that is not evident to calculate. Furthermore, the relationship between the bulk ultraviolet photoemission spectroscopy ionization energy and the oxidation potential of cyclic voltammetry requires a model in which it is assumed that the molecules form a thin film on the electrodes similar to that found in the bulk [91], which is also not easy to model theoretically. Because of these complications, we place our trust more firmly in gas phase results, where available, than in bulk phase results. Furthermore, as we shall see, reliable theoretical calculations are possible for the gas phase. Thus, once validated by comparison against gas phase data, we may use appropriate theoretical methodology to obtain a BDS of IPs and EAs.

Assessment of Our $G W$ Calculations Table 5 and Figure 7 show how various Green's function calculations compare to experimental data for predicting IPs. It is immediately obvious that the experimental molecular (gas phase) IPs are in much better agreement with the Green's function IPs than are experimental bulk (solution or thin film) IPs. This is, of course, to be expected on the basis of the above discussion. Of the different sets of Green's function IPs, our OVGF values are in the worst agreement with the experimental molecular IPs. As stated earlier (Section 3), we believe that this is simply because we were unable to carry out OVGF calculations with extensive basis sets. Both our $G W$ DOS and GW QP IPs agree as well, or better than, with the available experimental molecular IPs as do the $G W$ IPs taken from the literature. In the one case (PCBM) where a significant difference was observed between our $G W$ DOS and $G W$ QP IPs, it is the $G W$ QP IPs that are closer to the available experimental molecular value. This is because the DOS approach failed due to the HOMO level being smeared by the three nearly degenerate levels HOMO-1, HOMO-2 and HOMO-3.

Reliable experimental EA comparison data for molecules of interest in organic electronics is even harder to come across than for IPs. There are several reasons for this. Some are similar to those encountered with IPs, such as the difficulty of obtaining gas phase data. Some are particular to the experimental problem of obtaining electron addition rather than electron removal energies. Thus, our calculated EAs are vertical, while the experimental EAs are often best considered to be adiabatic, because of the experiments used to measure them. Furthermore, it must be remembered that that EAs tend to be smaller than IPs, except in the case of good electron acceptors, such as $\mathrm{C}_{60}$ and PCBM, whose EAs are expected to be so similar that they may be difficult to distinguish by theoretical calculations. Nevertheless, Table 6 and Figure 8 show that our $G W$ EAs also agree well with available experimental molecular (gas phase) data. 
Table 5. Comparison values of the first ionization potential for the medium-sized molecules from experiment and from quantum chemistry calculations. OVGF, outer-valence Green's function.

\begin{tabular}{|c|c|c|c|c|c|c|}
\hline \multirow[t]{4}{*}{ Molecule } & \multicolumn{2}{|c|}{ Experiment } & \multicolumn{4}{|c|}{ Green's Function } \\
\hline & \multirow[t]{3}{*}{ Bulk } & \multirow[t]{3}{*}{ Molecule } & \multirow[t]{3}{*}{ OVGF } & \multicolumn{3}{|c|}{$G W$} \\
\hline & & & & \multicolumn{2}{|c|}{ PW } & Literature \\
\hline & & & & $\operatorname{DOS}^{p}$ & $\mathbf{Q P}^{q}$ & \\
\hline \multirow[t]{2}{*}{ pentacene } & & $6.61^{e}$ & $5.78^{b}$ & 6.47 & 6.46 & $6.12^{d}$ \\
\hline & & & $6.27^{c}$ & & & \\
\hline DIP & $5.35^{m}$ & & $6.10^{b}$ & 6.91 & 6.89 & \\
\hline $\mathrm{H}_{2} \mathrm{Pc}$ & & $6.41^{f}$ & $5.81^{b}$ & 6.13 & 6.12 & $6.10^{d}$ \\
\hline PCBM & $5.87^{h}$ & $7.17^{n}$ & & 7.52 & 7.35 & \\
\hline $6 \mathrm{~T}$ & $4.7^{m}$ & & $5.86^{b}$ & 6.67 & 6.66 & \\
\hline DCV3T & $6.09^{a}$ & & $6.68^{b}$ & 7.79 & 7.78 & \\
\hline $\mathrm{C}_{60}$ & $6.45^{m}$ & $7.57^{g}$ & $7.43^{b}$ & 7.76 & 7.76 & $7.41^{d}$ \\
\hline rubrene & $5.92^{i}$ & $6.52^{i}$ & $5.60^{b}$ & 6.50 & 6.49 & $6.30^{l}$ \\
\hline \multirow[t]{2}{*}{ Molecule } & \multicolumn{4}{|c|}{ Koopmans' Theorem } & \multicolumn{2}{|c|}{$\Delta \mathrm{SCF}$} \\
\hline & $\mathrm{HF}$ & $\mathrm{B} 3 \mathrm{LYP}^{o}$ & DFTB & $\begin{array}{l}\text { SCC- } \\
\text { DFTB }\end{array}$ & $\mathrm{B} 3 L Y P^{o}$ & $\begin{array}{l}\text { SCC- } \\
\text { DFTB }\end{array}$ \\
\hline pentacene & $\begin{array}{l}5.91^{j} \\
6.09^{k}\end{array}$ & 4.59 & 5.38 & 4.97 & 5.93 & 6.81 \\
\hline DIP & $6.54^{j}$ & 5.12 & 5.73 & 5.35 & 6.32 & 6.99 \\
\hline $\mathrm{H}_{2} \mathrm{Pc}$ & $5.47^{j}$ & 4.93 & 5.14 & 4.96 & 6.05 & 6.49 \\
\hline PCBM & & 5.65 & 5.48 & 5.38 & 6.81 & 7.01 \\
\hline $6 \mathrm{~T}$ & $6.46^{j}$ & 4.82 & 5.17 & 4.79 & 5.88 & 6.22 \\
\hline DCV3T & $7.62^{j}$ & 6.01 & 5.40 & 5.64 & 7.10 & 7.21 \\
\hline $\mathrm{C}_{60}$ & $7.95^{j}$ & 5.98 & 5.67 & 5.67 & 7.22 & 7.41 \\
\hline rubrene & $6.08^{j}$ & 4.67 & 5.36 & 4.90 & 5.85 & 6.59 \\
\hline
\end{tabular}

${ }^{a}$ Estimated from cyclic voltammetry in $\mathrm{CH}_{2} \mathrm{Cl}_{2}$ [92]. ${ }^{b}$ Present work: OVGF/6-31G//B3LYP/6-31G**.

${ }^{c}$ Present work: OVGF/6-311++G**//B3LYP/6-31G**. ${ }^{d} G W$ from Table II of [36]. ${ }^{e}$ Gas-phase charge-stripping mass spectroscopy [93]. ${ }^{f}$ Gas-phase photoelectron spectroscopy (PES) [94]. ${ }^{g}$ From [95]. ${ }^{h}$ Thin-film ultraviolet photoemission spectroscopy (UPS) [96]. ${ }^{i}$ Gas and thin-film photoelectron spectroscopy (PES) [90]. ${ }^{j}$ Present work: HF/6-31G//B3LYP/6-31G**. ${ }^{k}$ Present work: HF/6-311++G**//B3LYP/6-31G**. ${ }^{l}$ From [97]. ${ }^{m}$ Ultraviolet photoemission spectroscopy (UPS) [98]. ${ }^{n}$ [99]. ${ }^{o}$ Present work: B3LYP/6-31G*//B3LYP/6-31G*. ${ }^{p}$ Present work: inspection of the density of states. ${ }^{q}$ Present work: solution of the quasiparticle equation. 


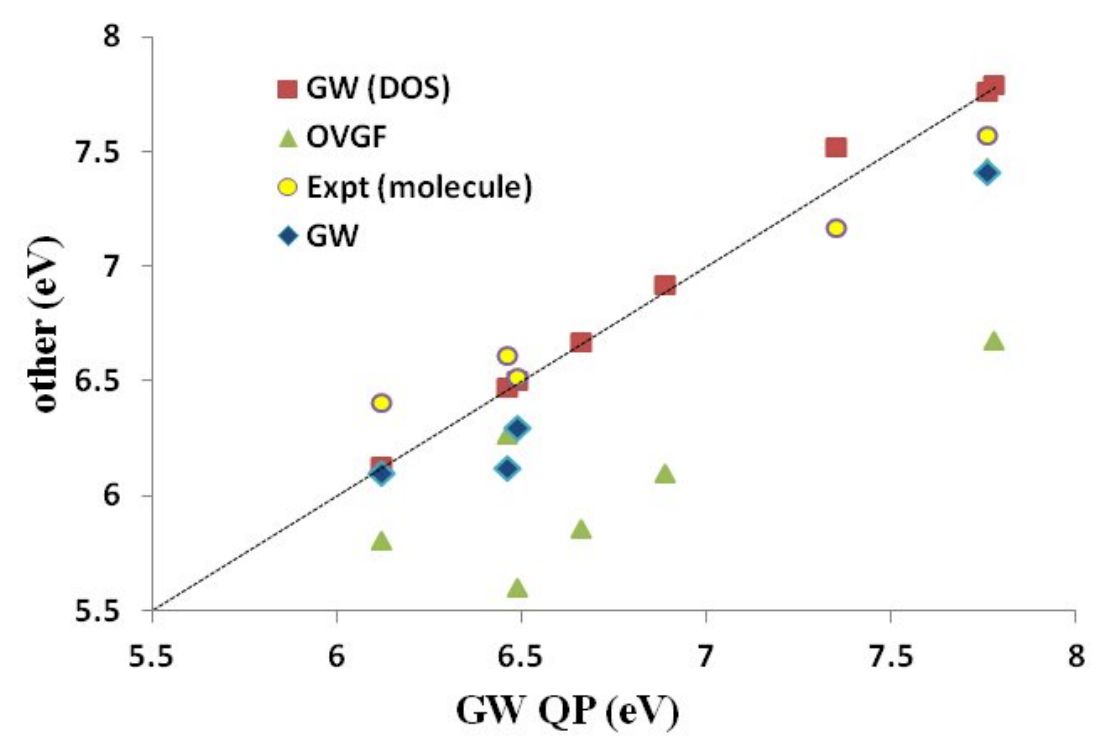

Figure 7. Correlation graph of data from Table 5 showing how experimental and Green's function IPs compare. The label " $G W$ " (as opposed to $G W$ DOS and $G W$ QP) is the calculated values taken from the literature (see Table 5). Units are in $\mathrm{eV}$. The $x$-axis corresponds to our $G W \mathrm{QP}$ calculations.

In conclusion, we feel justified in using our $G W$ QP values of IPs and EAs as a BDS for medium-sized molecules. We will use these to judge the quality of DFTB IPs and EAs.

Assessment of Our DFTB Calculations Figure 9 shows how DFTB and other approximate theoretical methods compare to our $G W$ QP IP BDS. Fitting data, including $\Delta y$ and $\Delta x$ values, are shown in Table 7. In general, the HF KT and the $\triangle \mathrm{SCF}$ methods give the best absolute agreement with the $G W$ QP results. Somewhat surprisingly, the HF KT IPs underestimate the GW QP IPs, though HF KT IPs typically overestimate experimental IPs for small molecules. The B3LYP $\triangle$ SCF values underestimate the GW QP IPs somewhat more than do the HF KT IPs. The SCC-DFTB $\triangle \mathrm{SCF}$ are perhaps closer in absolute values to the GW QP IPs, but the trend (slope $m$ in Table 7) is better for the B3LYP $\triangle$ SCF $(m=0.856)$ than for the SCC-DFTB $\triangle$ SCF $(m=0.520)$ method. As expected, the B3LYP KT, DFTB KT (the same as DFTB $\triangle$ SCF) and SCC-DFTB KT values are shifted down. The trends are best represented by the B3LYP KT ( $m=0.867)$ method, less so by the SCC-DFTB KT ( $m=0.504)$ method and very badly by the DFTB KT $(m=0.200)$ method. Focusing on $\Delta x$ as a measure of the predictive value, we see that the B3LYP method is most predictive ( $\Delta \mathrm{SCF} \Delta x=0.256 \mathrm{eV}, \mathrm{KT} \Delta x=0.270 \mathrm{eV}$ ), but that the SCC-DFTB method is still fairly good ( $\Delta \mathrm{SCF} \Delta x=0.489 \mathrm{eV}, \mathrm{KT} \Delta x=0.326 \mathrm{eV}$ ). Surprisingly, the SCC-DFTB KT approach seems to have somewhat more predictive power than does the SCC-DFTB $\triangle \mathrm{SCF}$ method, but we would not count on this always being the case. Of course, the original DFTB is not nearly as good $(\Delta x=0.982 \mathrm{eV})$. 
Table 6. Comparison values of the first electron affinity for the medium-sized molecules from the experiment and from quantum chemistry calculations.

\begin{tabular}{|c|c|c|c|c|c|c|}
\hline \multirow[t]{4}{*}{ Molecule } & \multicolumn{2}{|c|}{ Experiment } & \multicolumn{4}{|c|}{ Green's Function } \\
\hline & \multirow[t]{3}{*}{ Bulk } & \multirow[t]{3}{*}{ Molecule } & \multirow[t]{3}{*}{ OVGF } & \multicolumn{3}{|c|}{$G W$} \\
\hline & & & & \multicolumn{2}{|c|}{ PW } & Literature \\
\hline & & & & $\operatorname{DOS}^{p}$ & $\mathbf{Q P}^{q}$ & \\
\hline \multirow[t]{2}{*}{ pentacene } & & $1.35^{f}$ & $0.001^{c}$ & 0.985 & 0.981 & \\
\hline & & & $0.755^{d}$ & 0.941 & 0.953 & \\
\hline DIP & 2. ${ }^{g}$ & & $0.421^{c}$ & 1.31 & 1.30 & \\
\hline $\mathrm{H}_{2} \mathrm{Pc}$ & & & $1.063^{c}$ & 1.64 & 1.68 & \\
\hline PCBM & $5.96^{j}$ & $2.63^{n}$ & & 1.94 & 2.01 & \\
\hline $6 \mathrm{~T}$ & & $1.25^{m}$ & $-0.045^{c}$ & 0.413 & 0.422 & \\
\hline DCV3T & $3.90^{i}$ & & 1.201 & 1.85 & 1.86 & \\
\hline $\mathrm{C}_{60}$ & & $2.68^{n}$ & $1.486^{c}$ & 2.14 & 2.14 & \\
\hline rubrene & & & $-0.195^{c}$ & 0.839 & 0.822 & $1.88^{\circ}$ \\
\hline \multirow[t]{3}{*}{ Molecule } & \multicolumn{4}{|c|}{ Koopmans' Theorem } & \multicolumn{2}{|c|}{$\triangle \mathrm{SCF}$} \\
\hline & $\mathrm{HF}$ & $\mathrm{B} 3 \mathrm{LYP}^{e}$ & DFTB & SCC- & $\mathrm{B}^{2} \mathrm{LYP}^{e}$ & SCC- \\
\hline & & & & DFTB & & DFTB \\
\hline \multirow[t]{2}{*}{ pentacene } & $0.553^{k}$ & 2.38 & 4.05 & 3.63 & 1.05 & 1.80 \\
\hline & $-0.274^{l}$ & & & & & \\
\hline DIP & $-0.251^{k}$ & 2.59 & 4.14 & 3.75 & 1.39 & 2.10 \\
\hline $\mathrm{H}_{2} \mathrm{Pc}$ & $0.478^{k}$ & 2.83 & 3.94 & 3.65 & 1.71 & 2.08 \\
\hline PCBM & & 3.09 & 3.89 & 3.75 & 1.92 & 2.07 \\
\hline $6 \mathrm{~T}$ & $-0.519^{k}$ & 2.14 & 3.52 & 3.22 & 1.09 & 1.85 \\
\hline DCV3T & $0.792^{k}$ & 3.46 & 3.93 & 4.12 & 2.37 & 2.61 \\
\hline $\mathrm{C}_{60}$ & $0.679^{k}$ & 3.22 & 3.88 & 3.89 & 2.61 & 2.15 \\
\hline rubrene & $-0.957^{k}$ & 2.07 & 3.71 & 3.27 & 0.89 & 1.58 \\
\hline
\end{tabular}

$a$ Present work: inspection of the density of states. ${ }^{b}$ Present work: solution of the quasiparticle equation. ${ }^{c}$ Present work: OVGF/6-31G//B3LYP/6-31G**. $d$ Present work: OVGF/6-311++G**//B3LYP/6-31G**. ${ }^{e}$ Present work: B3LYP/6-31G*//B3LYP/6-31G*. ${ }^{f}$ Gas phase measurement using electron-transfer equilibria [100]. $g$ Thin film UPS and IPES [98]. ${ }^{h}$ [101]. ${ }^{i}$ Cyclic voltammetry in $\mathrm{CH}_{2} \mathrm{Cl}_{2}$ [92]. ${ }^{j}$ Solid-phase threshold electron affinity [102]. ${ }^{k}$ Present work: HF/6-31G. ${ }^{l}$ Present work: HF/6-311++G**. ${ }^{m}$ Gas-phase adiabatic electron affinity from photodetachment photoelectron spectroscopy [103]. ${ }^{n}$ Gas-phase adiabatic electron affinity from low-temperature photoelectron spectroscopy [104]. ${ }^{\circ} \mathrm{GW}$ calculation from [97]. 


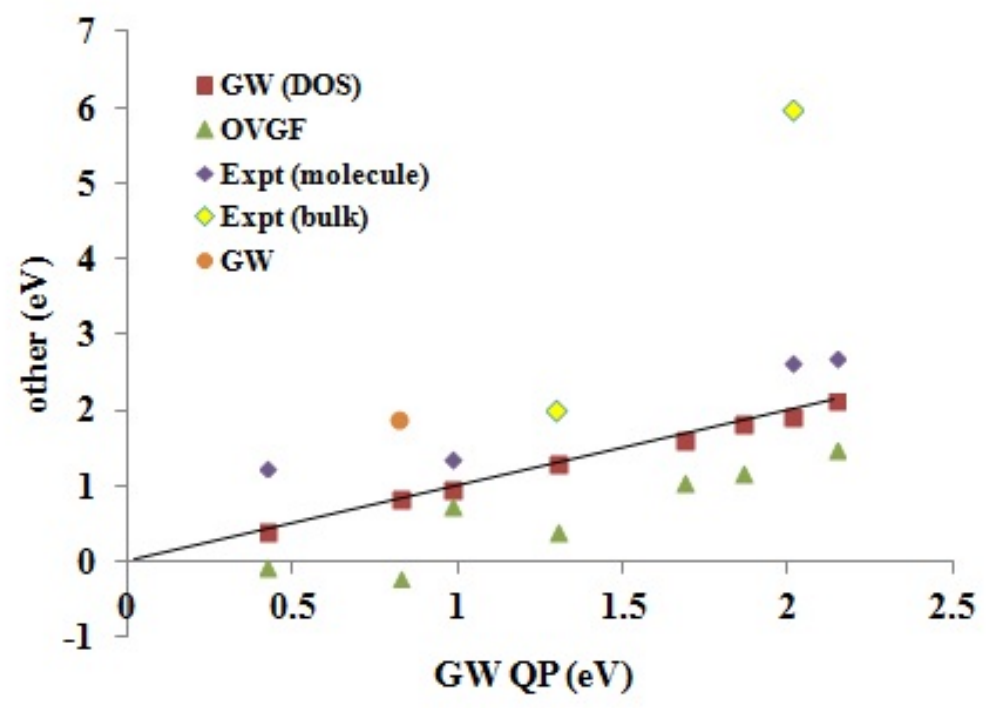

Figure 8. Correlation graph of data from Table 6 showing how experimental and Green's function EAs compare. The label " $G W$ " (as opposed to $G W$ DOS and $G W \mathrm{QP}$ ) is the calculated values taken from the literature (see Table 6).

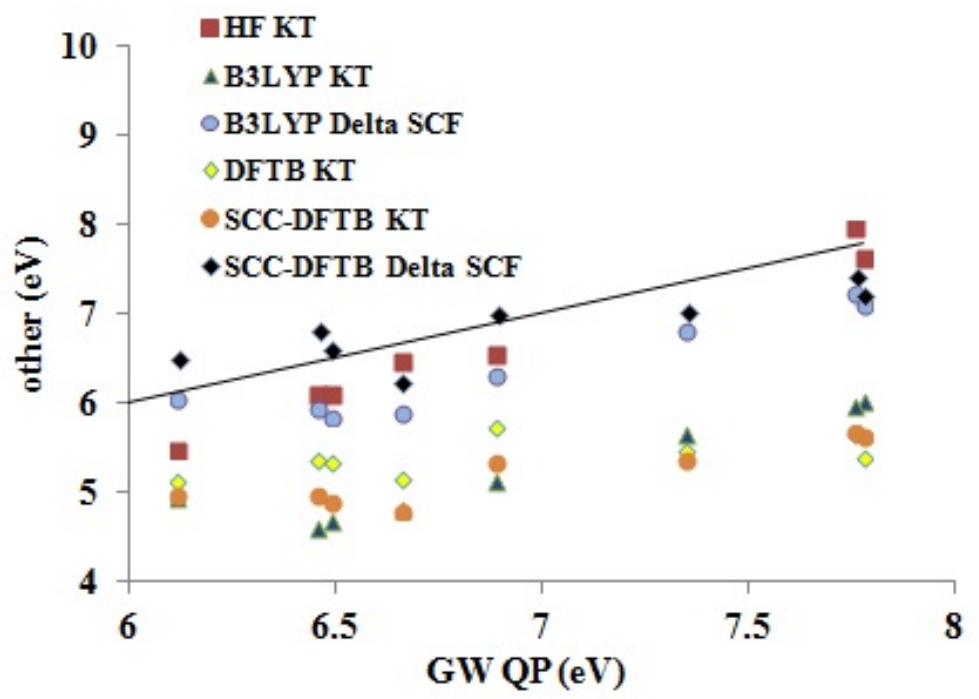

Figure 9. Correlation graph of data from Table 5 showing how the DFTB, SCC-DFTB and other methods compare to $G W$ QP IPs.

Figure 10 puts our results for medium-sized molecules in better perspective. In particular, it shows that the inversion procedure (Equation (40)) gives good results for the first IPs of both medium-sized and small molecules, but that a more sophisticated model than the present linear model would be required to maintain quantitative fitting for the binding energies of more tightly-bound electrons. Thus, our simple linear model seems to work well for band structure with $15 \mathrm{eV}$ or less binding energy, while a more elaborate analysis is in order for binding energies above $20 \mathrm{eV}$. 
Table 7. Fitting data for the first IPs of the medium-sized BDS molecules: $y=m x+b$, where $x$ is the $G W$ IP and $y$ is another theoretical IP, both in eV. HF, Hartree-Fock; OVGF, outer valence Green's function; LDA, local density approximation; DFTB, density-functional tight binding (without self-consistent charge); SCC-DFTB, self-consistent charge DFTB.

\begin{tabular}{|c|c|c|c|c|}
\hline Method & $m$ (Unitless) & $b(\mathbf{e V})$ & $\Delta y(\mathrm{eV})$ & $\Delta x(\mathbf{e V})$ \\
\hline \multicolumn{5}{|c|}{ Koopmans' Theorem } \\
\hline $\mathrm{HF}^{a}$ & 1.484 & -3.450 & 0.420 & 0.283 \\
\hline $\mathrm{HF}^{b}$ & 1.021 & -0.674 & 0.856 & 0.838 \\
\hline $\mathrm{HF}^{c}$ & 1.344 & -2.645 & 0.159 & 0.118 \\
\hline $\mathrm{OVGF}^{d}$ & 0.482 & 2.672 & 0.956 & 1.985 \\
\hline $\mathrm{OVGF}^{e}$ & 0.822 & 0.594 & 0.388 & 0.472 \\
\hline LDA & 0.782 & -0.024 & 0.361 & 0.461 \\
\hline B3LYP & 0.867 & -0.792 & 0.234 & 0.270 \\
\hline DFTB & 0.200 & 4.030 & 0.197 & 0.982 \\
\hline SCC-DFTB & 0.504 & 1.707 & 0.165 & 0.326 \\
\hline \multicolumn{5}{|c|}{$\Delta \mathrm{SCF}$} \\
\hline $\mathrm{HF}^{a}$ & 0.931 & -1.074 & 1.069 & 1.149 \\
\hline LDA & 0.644 & 2.472 & 0.318 & 0.493 \\
\hline B3LYP & 0.856 & 0.457 & 0.219 & 0.256 \\
\hline DFTB & 0.200 & 4.030 & 0.197 & 0.982 \\
\hline SCC-DFTB & 0.520 & 3.235 & 0.254 & 0.489 \\
\hline
\end{tabular}

a HF/6-31G*//HF/6-31G*, $\quad b \quad \mathrm{HF} / 6-31 \mathrm{G} / / \mathrm{B} 3 \mathrm{LYP} / 6-31 \mathrm{G}^{* *}$, except for PCBM, which was calculated at the HF/STO-3G//B3LYP/6-31G** level. ${ }^{c} \mathrm{HF} / 6-31 \mathrm{G} / / \mathrm{B} 3 \mathrm{LYP} / 6-31 \mathrm{G} * *$, excluding PCBM. ${ }^{d}$ OVGF/6-31G//B3LYP/6-31G**, except for PCBM, which was calculated at the OVGF/STO-3G//B3LYP/6-31G** level. ${ }^{e}$ OVGF/6-31G//B3LYP/6-31G**, excluding PCBM.

Figure 11 shows how the DFTB and other approximate methods compare to our $G W$ QP EA BDS. To get an idea of predictability, see Table 8. The HF KT EAs are expected to be an underestimate of the experimental values. Indeed, both relaxing the $(N+1)$-electron state and taking into account the greater correlation energy of the $(N+1)$-electron system compared to that of the $N$-electron system indicate that the HF KT EAs may be expected to be smaller than the true EAs. In this case, many of the HF KT EAs are negative and hence are unreliable unbound values. DFT KT EAs are expected to behave very differently, as the unoccupied DFT orbital energies may best be regarded as a first approximation on electronic excitation energies, which are typically smaller than EAs. This is seen in the overestimation of EAs by the DFTB KT and SCC-DFTB KT methods. As the B3LYP functional contains some admixture of HF exchange, it is normal that the B3LYP KT EAs lie somewhere in between the (SCC-)DFTB KT and HF KT methods. The $\triangle \mathrm{SCF}$ method results in a dramatic improvement for EAs in both the SCC-DFTB and B3LYP cases, with particularly good absolute EAs being produced by the B3LYP $\triangle$ SCF method. Table 8 suggests that the main difference between the LDA, B3LYP and SCC-DFTB KT and $\triangle$ SCF values is more in the value of the intercept $b$ than in a change in the slope $m$. This is consistent with 
the idea that the KT calculations contain a derivative discontinuity contribution not present in the $\triangle \mathrm{SCF}$ calculations. As commented on earlier, this is not true for the original non-SCC DFTB method where the KT and $\triangle \mathrm{SCF}$ calculations always give the same result. Furthermore, comparing Tables 7 and 8 , we see that the main difference between the IP and EA B3LYP KT and SCC-DFTB KT fits is also in the intercept $b$, rather than the slope $m$, also consistent with an important derivative discontinuity effect. Interestingly, the IP and EA B3LYP $\triangle$ SCF fits show only a small shift of the intercept $b$, while the IP and EA SCC-DFTB $\triangle$ SCF fits show a more distinct shift.

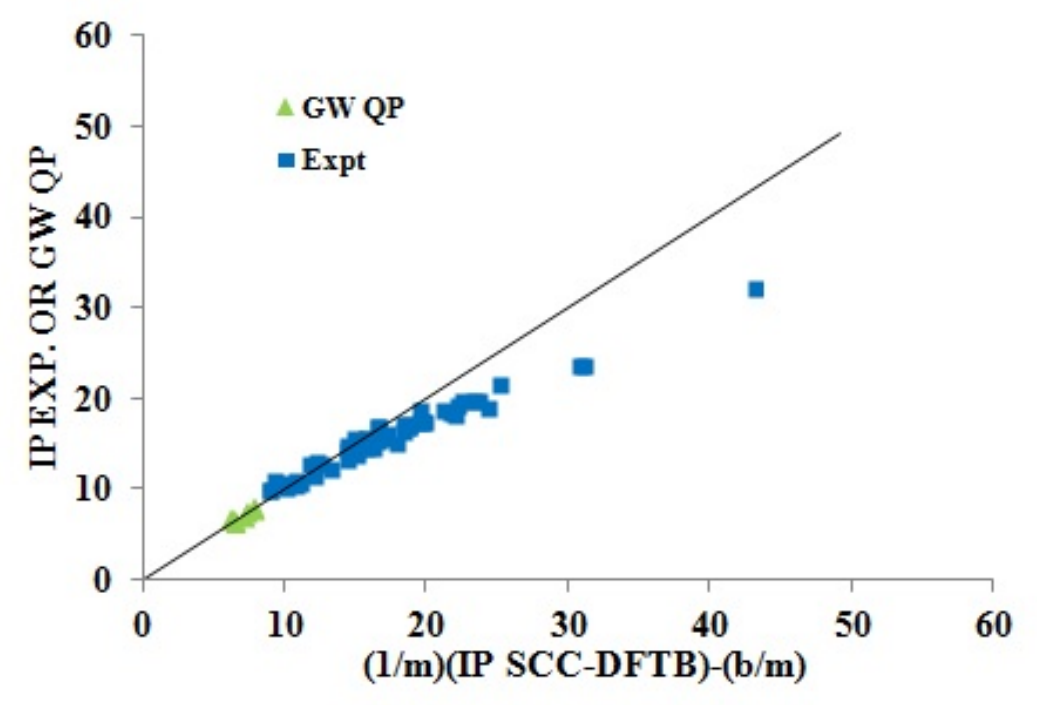

Figure 10. Inversion plot showing how fitting parameters obtained for the SCC-DFTB KT IPs and medium-sized molecules predict the GW QP IPs of medium-sized molecules and the experimental IPs of small molecules.

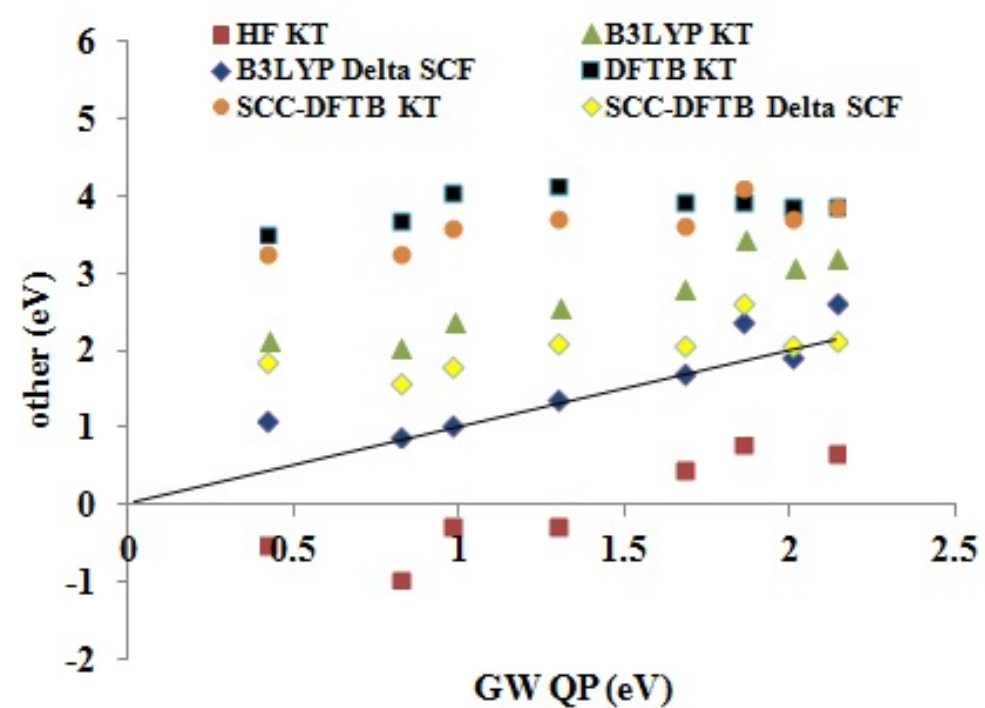

Figure 11. Correlation graph of data from Table 5 showing how the DFTB, SCC-DFTB and other methods ( $y$-axis, eV) compare with $G W \mathrm{QP}(x$-axis, eV) EAs. 
Table 8. Fitting data for first EAs of the medium-sized benchmark dataset (BDS) molecules: $y=m x+b$, where $x$ is the $G W$ EA and $y$ is another theoretical EA, both in eV. HF, Hartree-Fock; OVGF, outer valence Green's function; LDA, local density approximation; DFTB, density-functional tight binding (without self-consistent charge); SCC-DFTB, self-consistent charge DFTB.

\begin{tabular}{lcccc}
\hline Method & $\boldsymbol{m}$ (Unitless) & \multicolumn{1}{c}{$\boldsymbol{b}(\mathbf{e V})$} & $\boldsymbol{\Delta} \boldsymbol{y}(\mathbf{e V})$ & $\boldsymbol{\Delta} \boldsymbol{x}(\mathbf{e V})$ \\
\hline $\mathrm{HF}^{a}$ & -0.817 & 1.772 & 0.181 & 0.222 \\
$\mathrm{HF}^{b}$ & 1.455 & -1.695 & 0.860 & 0.591 \\
$\mathrm{HF}^{c}$ & 0.971 & -1.289 & 0.344 & 0.354 \\
$\mathrm{OVGF}^{d}$ & 1.183 & -0.777 & 0.475 & 0.402 \\
OVGF $^{e}$ & 0.948 & -0.578 & 0.487 & 0.513 \\
LDA $^{c}$ & 0.801 & 2.815 & 0.197 & 0.246 \\
B3LYP & 0.769 & 1.645 & 0.218 & 0.284 \\
DFTB & 0.151 & 3.672 & 0.195 & 1.298 \\
SCC-DFTB & 0.407 & 3.089 & 0.186 & 0.458 \\
\hline \multicolumn{5}{c}{$\Delta \mathrm{SCF}$} \\
\hline HF & \\
LDA & 1.020 & -0.833 & 0.460 & 0.451 \\
B3LYP & 0.917 & 1.107 & 0.303 & 0.331 \\
DFTB & 0.926 & 0.330 & 0.317 & 0.342 \\
SCC-DFTB & 0.150 & 3.672 & 0.195 & 1.298 \\
\hline
\end{tabular}

a $\mathrm{HF} / 6-31 \mathrm{G}^{*} / / \mathrm{HF} / 6-31 \mathrm{G}^{*}, \quad b \quad \mathrm{HF} / 6-31 \mathrm{G} / / \mathrm{B} 3 \mathrm{LYP} / 6-31 \mathrm{G}^{* *}$, except for PCBM , which was calculated at the HF/STO-3G//B3LYP/6-31G** level. ${ }^{c} \mathrm{HF} / 6-31 \mathrm{G} / / \mathrm{B} 3 \mathrm{LYP} / 6-31 \mathrm{G}^{* *}$, excluding PCBM. ${ }^{d}$ OVGF/6-31G//B3LYP/6-31G**, except for PCBM, which was calculated at the OVGF/STO-3G//B3LYP/6-31G** level. ${ }^{e}$ OVGF/6-31G//B3LYP/6-31G**, excluding PCBM.

\section{Conclusions}

As emphasized, for example, by the Shockley diode model presented in the Appendix and perhaps even better by the Shockley-like model developed in [5] specifically for organic solar cells, it is important to be able to understand the underlying phenomenology of organic photovoltaics at the atomistic level. However, these are complex systems that can benefit from both molecular and solid-state theory and that ultimately are likely to require hybrid approaches, which are somewhere between what is now standard for molecules and what is now standard for inorganic solids. It is a problem, in fact, at the "nanoscale interface" between these two ideological paradigms, which is likely to require the modeling the electronic structure and dynamics of ensembles of objects on the order of 1-100 nm in size. As this is beyond the reach of most first principles methods, we have decided to adopt the semi-empirical DFTB approach as a way to try to extend first principles accuracy to larger systems. Naturally, this also requires us to accept some loss of accuracy whose magnitude we have tried to evaluate for IPs and EAs of small molecules and of medium-sized molecules of interest for organic electronics. 
This paper has presented a test of the accuracy of DFTB and especially of third-order SCC-DFTB. It has been possible to determine this for larger molecules than is usually the case because of our access to the recently-developed $G W$ methodology, which itself has been checked against available experimental values. The final result is that DFTB3 with the $3 \mathrm{ob}$ parameter set is found to give the first IPs with an accuracy of $\pm 0.489 \mathrm{ev} / \pm 0.326 \mathrm{eV}$ with the SCC-DFTB $\triangle \mathrm{SCF} / \mathrm{KT}$ approaches. To judge from Figure 10, lower IPs may also be determined with a similar accuracy using the SCC-DFTB KT method. The first EAs are found to be accurate to $\pm 0.740 \mathrm{eV} / \pm 0.458 \mathrm{eV}$ with the SCC-DFTB $\Delta \mathrm{SCF} / \mathrm{KT}$ approaches. We find this quite encouraging and expect that one should still be able to answer quite a few well-posed questions in organic electronics with this level of accuracy. There is a caveat, however: the reader should keep in mind that results will change if a different implementation of DFTB and/or another parameter set are used.

A perhaps more important observation is that, while it is possible to optimize a semi-empirical method explicitly for IPs and EAs, the parameters in the DFTB method have not been optimized with this end in mind, but rather with the idea of being a well-balanced approximation to DFT. Thus, we repeatedly find that DFTB is behaving like DFT rather than, say, a correlated HF-based ab initio method. Moreover, though the SCC-DFTB formalism differs significantly in details from the self-consistent solution of the DFT equations, we do find that (much like in DFT) the best way to calculate IPs and EAs is to use the $\triangle$ SCF method with SCC-DFTB. However, much like in regular DFT calculations, SCC-DFTB KT IPs are also found to be a reasonable way to estimate the first several IPs simultaneously and, hence, to obtain the molecular analogue of band spectra. Such a similarity with DFT, together with the capacity to go to larger and more complex systems, makes DFTB particularly attractive to us as we wish to go well beyond merely studying IPs and EAs to using DFTB to study the details of photochemical processes at the donor-acceptor interface in organic solar cells, as all indications point to the details of polaron pair dissociation as being a key determining step to organic photocell performance [5]. Of course, where possible, we will continue to check our DFTB results against experiment and against good first-principles computational methods as we go along.

\section{Acknowledgments}

This work is supported, in part, by the ORGAVOLT (ORGAnic solar cell VOLTage by numerical computation) Grant ANR-12-MONU-0014-02 of the French Agence Nationale de la Recherche (ANR) 2012 Programme Modèles Numériques. Mathias P. Ljungberg, Peter Koval and Daniel Sánchez-Portal acknowledge support from the Deutsche Forschungsgemeinschaft (DFG) through the SFB (Sonderforschungsbereich) 1083 project, the Spanish MINECO (Ministerio de Economía y Competitividad) MAT2013-46593-C6-2-P project and the Euroregion Aquitaine-Euskadi program and support from the Basque Departamento de Educación, UPV/EHU (University of the Basque Country) (Grant No. IT-756-13). Peter Koval acknowledges financial support from the Fellows Gipuzkoa program of the Gipuzkoako Foru Aldundia through the FEDER funding scheme of the European Union. Ala Aldin M. H. M. Darghouth would like to thank the French and Iraq governments for support through a Campus France scholarship. Walid Taouali would like to acknowledge support from the government of Tunisia. Grenoble Centre d'Expérimentation du Calcul Intensif en Chimie (CECIC) computing resources are gratefully acknowledged, as is computing help from 
Pierre Girard. Mark E. Casida would like to acknowledge a helpful discussion with Kuntheak Kheng. Mark E. Casida and Ala Aldin M. H. M. Darghouth would like to acknowledge helpful discussions with Mathias Rapacioli. We would also like to thank Thomas A. Niehaus for his comments on an earlier version of this manuscript and for calling our attention to the preprint [14].

\section{Author Contributions}

Calculations were carried out by Ala Aldin M. H. M. Darghouth (DFTB, DFT, OVGF), Walid Taouali (DFT) and Peter Koval ( $G W$ ) under the direction of Mark E. Casida (Ala Aldin M. H. M. Darghouth), Kamel Alimi (Walid Taouali) and Daniel Sánchez-Portal (Mathias P. Ljungberg and Peter Koval). Significant programming in the mbpt_lcao code was done by Dietrich Foerster, Daniel Sánchez-Portal, Mathias P. Ljungberg and Peter Koval. The bulk of the manuscript writing was done by Mark E. Casida with significant contributions from Ala Aldin M. H. M. Darghouth, Walid Taouali, Mathias P. Ljungberg and Peter Koval. All authors have read and approved the final manuscript.

\section{Conflicts of Interest}

The authors declare no conflict of interest.

\section{A. Brief Review of Shockley Diode Model's for Organic Solar Cells}

Though some progress has been made in recent years on developing an improved physical model for organic solar cells [5,6], the most frequent model used to describe a simple bilayer "Tang" cell involving touching layers of donor (D) and acceptor (A) molecules [105] continues to be the Shockley diode model (pp. 115-117 of [106]) familiar from the semiconductor device literature. As in Figure A1, the A-D interface is identified with the p-n layer in the Shockley theory. Here, $V$ is the applied voltage (represented by the battery symbol) and $R$ is the resistance in series with the applied voltage.

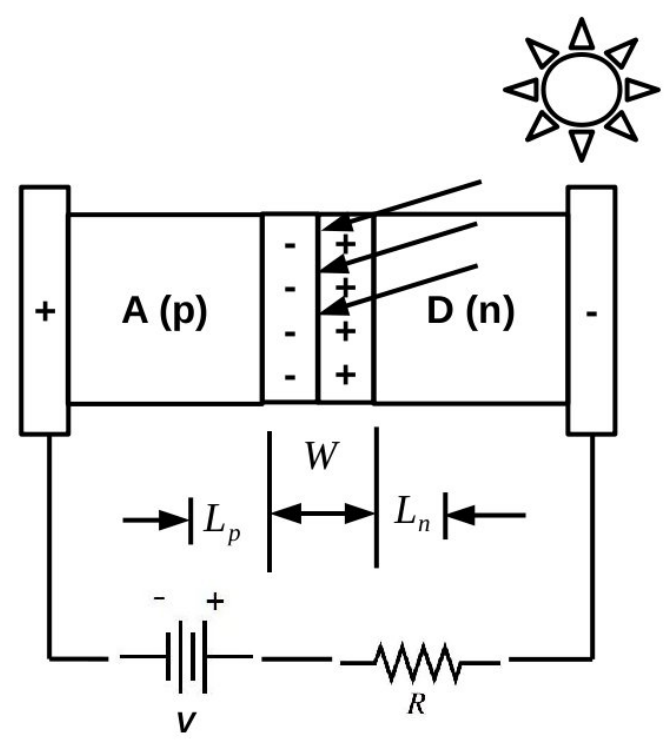

Figure A1. Schematic of an organic solar cell as a Shockley diode. 
According to the depletion approximation ([107], pp. 159-185), charge transfer already occurs in the unperturbed system (in the dark with $V=0$ and the current $I=0$ ), creating a dipole bilayer depletion region. Analysis of the current in this region ([107], pp. 462-470) leads to the diode equation,

$$
I=I_{L}+I_{0}\left[1-\exp \left(\frac{V+R I}{n V_{T}}\right)\right]
$$

where $V_{T}=k T / e$ is the "thermal voltage" and $n$ is the ideality factor ( $n \neq 1$ is an empirical correction to include non-ideal charge generation and recombination effects ([107], p. 186)). The short-circuit voltage is $I_{s c}=I_{L}$, while the open-circuit voltage is,

$$
V_{o c}=n V_{T} \ln \left(1+\frac{I_{L}}{I_{0}}\right)
$$

This involves the reverse bias saturation current,

$$
\lim _{V \rightarrow-\infty}\left(I-I_{L}\right)=I_{0}=e A\left(\frac{D_{p} p_{n}}{L_{p}}+\frac{D_{n} n_{p}}{L_{n}}\right)
$$

where $A$ is the area of the solar cell cross-section, $D_{p}$ is the diffusion coefficient for the positive charge carriers, $D_{n}$ is the diffusion coefficient for the negative charge carriers, $L_{p}$ and $L_{n}$ are the corresponding diffusion lengths, $n_{p}$ is the density of negative carriers in the acceptor half of the solar cell and $p_{n}$ is the density of positive carriers in the donor half of the solar cell. The short-circuit current,

$$
I_{s c}=e \alpha J_{p h}\left(L_{p}+L_{n}+W\right) A
$$

involves the width $W$ of the depletion region, the photon flux $J_{p h}$ and the absorption coefficient $\alpha$, which is defined in terms of the variation of optical power in the material, $P_{o p}(x)=P_{o p}(0) \exp (-\alpha x)$ as a function of the depth, $x$ of light penetration. The diode model presented above could be made more elaborate, but it already captures much of the fundamental physics and is sufficient for the present purposes. The diode equation also gives a good empirical fit to experiment in many cases. However, it has been criticized on the grounds that the physical quantities used to derive the Shockley model are not the most appropriate ones to describe an organic solar cell and that some phenomena require a different derivation, which is more closely tied to the fundamental physics of organic solar cells $[5,6]$, and that the Shockley model cannot describe certain phenomena, such as the S-shaped current-voltage curves sometimes observed in organic solar cells [5,6,108,109]. Either in the context of the Shockley model or, better yet, in terms of some improved model, it is tempting to try to calculate first principles parameters for use in the diode equation for comparison against experiment and, in particular, to seek a better understanding of how charge separation occurs near the donor-acceptor interface, both in the dark and when illuminated.

One thing that is conspicuously missing from the above theory is the notion of an energy gap, $E_{g}$. This may be recovered by applying the detailed balance assumption [110] (i) that all absorbed photons create an electron-hole pair and (ii) that every photon whose energy is greater than the fundamental gap $E_{g}$ is absorbed. In the present case, this leads to the intuitively appealing result [111] that,

$$
V_{o c} \leq E_{g}
$$


with near equality for ideal solar cells. While $V_{o c} \approx E_{g}$ is certainly much easier to calculate than using Equation (A2), we must remember that this is only an ideal limit. Furthermore, the basic theoretical argument does not specify the nature of the gap, only that every photon is absorbed whose energy exceeds that gap. The nature of this gap in real organic solar cells has been a matter of some discussion. Thus, an early paper of Brabec et al. argued that the difference between the highest occupied molecular orbital (HOMO) energy of the donor and the lowest unoccupied molecular orbital (LUMO) energy of the acceptor was the dominant determining factor for the gap in organic solar cells [112], in contradiction with earlier work, which indicated the importance of the difference of the work functions of the electrodes [113,114]. This seems to have been resolved by latter work [115] showing that for ohmic contacts,

$$
V_{o c}=\frac{1}{e}\left(\epsilon_{\mathrm{HOMO}}^{D}-\epsilon_{\mathrm{LUMO}}^{A}\right)+\Delta V_{b}
$$

where $\mathrm{IP}_{D}=-\epsilon_{\mathrm{HOMO}}^{D}$ is the electron removal energy of the donor, $\mathrm{EA}_{A}=-\epsilon_{\mathrm{LUMO}}^{A}$ is the electron attachment energy of the acceptor and $\Delta V_{b}$ is a correction term for band bending at the electrodes, which can presumably be made arbitrarily small for ohmic contacts with small band offsets. Equation (A6) is written in the language of band theory. Let us emphasize that it is simply an equation involving the donor ionization potential and the acceptor electron affinity,

$$
V_{o c}=\frac{1}{e}\left(\mathrm{EA}_{A}-\mathrm{IP}_{D}\right)+\mathrm{constant}
$$

where the "constant" is presumably a function of the nature of the electrode junctions (see also [116]).

\section{References}

1. Sze, S.M.; Ng, K.K. Physics of Semiconductor Devices, 3rd ed.; Wiley: Hoboken, NJ, USA, 2007.

2. Nagel, L.W.; Pederson, D.O. SPICE (Simulation Program with Integrated Circuit Emphasis), Memorandum No. ERL-M382, University of California, Berkeley, April 1973; SPICE2: A Computer Program to Simulate Semiconductor Circuits, Memorandum No. ERL-M520; Nagel, L.W., Ed.; University of California: Berkeley, CA, USA, 1975.

3. Harada, K.; Werner, A.G.; Pfeiffer, M.; Bloom, C.J.; Eliott, C.M.; Leo, K. Organic homojunction diodes with a high built-in potential: Interpretation of the current-voltage characteristics by a generalized Einstein relation. Phys. Rev. Lett. 2005, 94, 036601.

4. Vaynzof, Y.; Preezant, Y.; Tessler, N. Current voltage relation of amorphous materials based pn diodes-The effect of degeneracy in organic polymers/molecules. J. Appl. Phys. 2009, $106,084503$.

5. Giebink, N.C.; Wiederrecht, G.P.; Waslielewski, M.R.; Forrest, S.R. Ideal diode equation for organic heterojunctions. I. Derivation and application. Phys. Rev. B 2010, 82, 155305.

6. Giebink, N.C.; Lassiter, B.E.; Wiederrecht, G.P.; Wasielewski, M.R.; Forrest, S.R. Ideal diode equation for organic heterojunctions. II. The role of polaron pair recombination. Phys. Rev. B 2010, 82, 155306.

7. Tripathi, D.C.; Mohapatra, Y.N. Ideal organic homojunction diode obtained using controlled alignment of localized density of states across doped/undoped interface. Org. Electron. 2012, $13,1680-1685$. 
8. Dwivedi, A.D.D. Numerical Simulation and Spice Modeling of Organic Thin Film Transistors (OTFTs). Int. J. Adv. Appl. Phys. Res. 2014, 1, 14-21.

9. Müllen, K.; Scherf, U. (Eds.) Organic Light-Emitting Devices; Springer-Verlag: Berlin, Germany, 1990.

10. Yu, G.; Gao, J.; Hummelen, J.C.; Wudl, F.; Heeger, A.J. Polymer Photovoltaic Cells: Enhanced Efficiencies via a Network of Internal Donor-Acceptor Heterojunctions. Science 1995, 270, 1789-1791 .

11. Huang, Y.S.; Westenhoff, S.; Avilov, I.; Sreearunothai, P.; Hodgkiss, J.M.; Deleener, C.; Friend, R.H.; Beljonne, D. Electronic structures of interfacial states formed at polymeric semiconductor heterojunctions. Nat. Mater. 2008, 7, 483-489.

12. Dou, L.; You, J.; Hong, Z.; Xu, Z.; Li, G.; Street, R.A.; Yang, Y. 25th anniversary article: A decade of organic/polymer photovoltaic research. Adv. Mater. 2013, 25, 6642-6671.

13. Scholz, R.; Luschtinetz, R.; Seifert, G.; Jägerler-Hoheisel, T.; Körner, C.; Leo, K.; Rapacioli, M. Topical Review: Quantifying charge transfer energies at donor-acceptor interfaces in small-molecule solar cells with constrained DFTB and spectroscopic methods. J. Phys. 2013, 25, 473201.

14. Lutsker, V.; Aradi, B.; Niehaus, T.A. Implementation and benchmark of a long-range corrected functional in the density functional based tight-binding method. J. Chem. Phys. 2015, 143, 184107.

15. Niehaus, T.; Salla, F.D. Range separated functionals in the density functional based tight-binding method: Formalism. Phys. Stat. Solidi B 2012, 249, 237-244.

16. Humeniuk, A.; Mitrić, R. Long-range correction for tight-binding TD-DFT. J. Chem. Phys. 2015, $143,134120$.

17. Hedin, L. New Method for Calculating the One-Particle Green's Function with Application to the Electron-Gas Problem. Phys. Rev. 1965, 139, doi:10.1103/PhysRev.139.A796.

18. Onida, G.; Reining, L.; Rubio, A. Electronic excitations: Density-functional versus many-body Green's-function approaches. Rev. Mod. Phys. 2002, 74, doi:10.1103/RevModPhys.74.601.

19. Foerster, D.; Koval, P.; Sanchez-Portal, D. An $\mathcal{O}\left(N^{3}\right)$ implementation of Hedin's $G W$ approximation for molecules. J. Chem. Phys. 2011, 135, 074105.

20. Pilar, F. Elementary Quantum Chemistry; McGraw-Hill Book Co.: New York, NY, USA, 1968.

21. Parr, R.G.; Yang, W. Density-Functional Theory of Atoms and Molecules; Oxford University Press: New York, NY, USA, 1976.

22. Dreizler, R.M.; Gross, E.K.U. Density Functional Theory: An Approach to the Quantum Many-Body Problem; Springer-Verlag: New York, NY, USA, 1990.

23. Koch, W.; Holthausen, M.C. A Chemist's Guide to Density Functional Theory; Wiley-VCH: New York, NY, USA, 2000.

24. Burke, K. Perspective on density functional theory. J. Chem. Phys. 2012, 136, 150901.

25. Becke, A.D. Perspective: Fifty years of density-functional theory in chemical physics. J. Chem. Phys. 2014, 140, 18A301.

26. Whittleton, S.R.; Vazquez, X.A.S.; Isborn, C.M.; Johnson, E.R. Density-functional errors in ionization potentials with increasing system size. J. Chem. Phys. 2015, 142, 184106. 
27. Danovich, D. Green's function methods for calculating ionization potentials, electron affinities, and excitation energies. Comput. Molec. Sci. 2011, 1, 377-387.

28. Von Niessen, W.; Schirmer, J.; Cederbaum, L.S. Computational methods for the one-particle Green's function. Comput. Phys. Rep. 1984, 1, 57-125.

29. Casida, M.E.; Chong, D.P. Physical interpretation and assessment of the Coulomb-hole and screened-exchange approximation for molecules. Phys. Rev. A 1989, 40, 4837-4848.

30. Casida, M.E.; Chong, D.P. Erratum: "Physical interpretation and assessment of the Coulomb-hole and screened-exchange approximation for molecules". Phys. Rev. A 1991, 44, 6151.

31. Casida, M.E.; Chong, D.P. Simplified Green-function approximations: Further assessment of a polarization model for second-order calculation of outer-valence ionization potentials in molecules. Phys. Rev. A 1991, 44, 5773-5783.

32. Onida, G.; Reining, L.; Godby, R.W.; Del Sole, R.; Andreoni, W. Ab initio calculations of the quasiparticle and absorption spectra of clusters: The sodium tetramer. Phys. Rev. Lett. 1995, $75,818-821$.

33. Hu, C.; Chong, D.P.; Casida, M.E. The parameterized second-order Green function times screened interaction (pGW2) approximation for calculation of outer valence ionization potentials. $J$. Electron Spectr. 1997, 85, 39.

34. Shigeta, Y.; Ferreira, A.M.; Zakrzewski, V.G.; Ortiz, J.V. Electron propagator calculations with Kohn-Sham reference states. Int. J. Quant. Chem. 2001, 85, 411-420.

35. Hahn, P.H.; Schmidt, W.G.; Bechstedt, F. Molecular electronic excitations from a solid-state approach. Phys. Rev. B 2005, 72, 245425.

36. Blase, X.; Attaccalite, C.; Olevano, V. First-principles $G W$ calculations for fullerenes, porphyrins, phtalocyanine, and other molecules of interest for organic photovoltaic applications. Phys. Rev. B 2011, 83, 115103.

37. Casida, M.E. Generalization of the Optimized Effective Potential Model to Include Electron Correlation: A Variational Derivation of the Sham-Schlüter Equation for the Exact Exchange-Correlation Potential. Phys. Rev. A 1995, 51, doi:10.1103/PhysRevA.51.2005.

38. Duffy, P.; Chong, D.; Casida, M.E.; Salahub, D.R. Assessment of Kohn-Sham Density-Functional Orbitals as Approximate Dyson Orbitals for the Calculation of Electron-Momentum-Spectroscopy Scattering Cross Sections. Phys. Rev. A 1994, 50, 4707.

39. Brion, C.E.; Cooper, G.; Zheng, Y.; Litvinyuk, I.V.; McCarthy, I.E. Imaging of orbital electron densities by electron momentm spectroscopy-A chemical interpretation of the binary (e,2e) reaction. Chem. Phys. 2001, 270, 13-30.

40. Hamel, S.; Duffy, P.; Casida, M.E.; Salahub, D.R. Kohn-Sham orbitals and orbital energies: Fictitious constructs but good approximations all the same. J. Electr. Spectr. Related Phenom. 2002, 123, 345-363.

41. Hamel, S.; Casida, M.E.; Salahub, D.R. Exchange-only optimized effective potential for molecules from resolution-of-the-identity techniques: Comparison with the local density approximation, with and without asymptotic correction. J. Chem. Phys. 2002, 116, 8276-8291.

42. Godby, R.W.; Schlüter, M.; Sham, L.J. Self-energy operators and exchange-correlation potentials in semiconductors. Phys. Rev. B 1988, 37, 10159. 
43. Levine, Z.H.; Allan, D.C. Linear optical response in silicon and germanium including self-energy effects. Phys. Rev. Lett. 1989, 63, 1719.

44. Gonze, X.; Lee, C. Dynamical matrices, Born effective charges, dielectric permittivity tensors and interatomic force constants from density-functional perturbation theory. Phys. Rev. B 1997, $55,10355$.

45. Hückel, E. Quantentheoretische Beiträge zum Benzolproblem. Z. Physik 1931, 70, 204-286.

46. Hückel, E. Quantentheoretische Beiträge zum Benzolproblem II. Quantentheorie der induzierten Polaritäten. Z. Physik 1931, 72, 310.

47. Hückel, E. Quantentheoretische Beiträge zum Problem der aromatischen und ungesättigten Verbindungen. III. Z. Physik 1932, 76, 628.

48. Slater, J.C.; Koster, G.F. Simplified LCAO Method for the Periodic Potential Problem. Phys. Rev. 1954, 94, 1498.

49. Hoffmann, R. An extended Hückel theory. I. Hydrocarbons. J. Chem. Phys. 1963, 39, 1397-1412.

50. Hoffmann, R. An extended Hückel theory. II. $\sigma$ orbitals in the azines. J. Chem. Phys. 1964, 40, 2745.

51. Hoffmann, R. An extended Hückel theory. III. Compounds of boron and nitrogen. J. Chem. Phys. 1964, 40, 2474-2480.

52. Hoffmann, R. An extended Hückel theory. IV. Carbonium ions. J. Chem. Phys. 1964, 40, 2480-2488.

53. Porezag, D.; Frauenheim, T.; Köhler, T.; Seifert, G.; Kaschner, R. Construction of tight-binding-like potentials on the basis of density-functional theory: Application to carbon. Phys. Rev. B 1995, 51, 12947.

54. Elstner, M. Weiterentwicklung Quantenmechanischer Rechenverfahren für organische Moleküle und Polymere. Ph.D. Thesis, Universität-Gesamthochschule Paderborn, Paderborn, Germany, 1998. (In German)

55. Frauenheim, A.; Seifert, G.; Elstner, M.; Hajnal, Z.; Jungnickel, Z.; Porezag, D.; Suhai, S.; Scholz, R. A self-consistent charge density-functional based tight-binding method for predictive materials simulations in physics, chemistry, and biology. Phys. Stat. Sol. B 2000, 217, 41-62.

56. Koskinen, P.; Mäkinen, V. Density-functional tight-binding for beginners. Comput. Mater. Sci. 2009, 47, 237-253.

57. Bredow, T.; Jug, K. Theory and range of modern semiempirical molecular orbital methods. Theor. Chem. Acc. 2005, 113, 1-14.

58. Thiel, W. Semiempirical Quantum-Chemical Methods in Computational Chemistry. In Theory and Applications of Computational Chemistry: The First Forty Years; Dykstra, C.; Frenking, G., Kim, K., Scuseria, G., Eds.; Elsevier: Amsterdam, The Netherlands, 2005; Chapter 21, pp. 559-580.

59. Hohenberg, P.; Kohn, W. Inhomogeneous electron gas. Phys. Rev. 1964, 136, B864.

60. Kohn, W.; Sham, L.J. Self-consistent equations including exchange and correlation effects. Phys. Rev. 1965, 140, A1133. 
61. Elstner, M.; Porezag, D.; Jungnickel, G.; Elsner, J.; Haugk, M.; Frauenheim, T.; Suhai, S.; Seifert, G. Self-consistent-charge density-functional tight-binding method for simulations of complex materials properties. Phys. Rev. B 1998, 58, 7260.

62. Gaus, M.; Cui, Q.; Elstner, M. DFTB3: Extension of the Self-Consistent-Charge Density-Functional Tight-Binding Method (SCC-DFTB). J. Chem. Theory Comput. 2011, 7, 931-948.

63. Pariser, R. An improvement on the $\pi$-electron approximation in LCAO MO theory. J. Chem. Phys. 1953, 21, 568-569.

64. DFTB+. Available online: http://www.dftb-plus.info/ (accessed on 23 November 2015).

65. Frisch, M.J.; Trucks, G.W.; Schlegel, H.B.; Scuseria, G.E.; Robb, M.A.; Cheeseman, J.R.; Scalmani, G.; Barone, V.; Mennucci, B.; Petersson, G.A.; et al. Gaussian 09 Revision D.01; Gaussian Inc.: Wallingford, CT, USA, 2009.

66. Soler, J.M.; Artacho, E.; Gale, J.D.; García, A.; Junquera, J.; Ordejón, P.; Sánchez-Portal, D. The SIESTA method for ab initio order- $N$ materials simulation. J. Phys.: Condens. Matter 2002, $14,2745$.

67. Official Web Page for mbpt_lcao. Available online: http://mbpt-domiprod.wikidot.com/ (accessed on 30 November 2015).

68. Hehre, W.J.; Radom, L.; von R. Schleyer, P.; Pople, J. Ab Initio Molecular Orbital Theory; Wiley: Hoboken, NJ, USA, 1986.

69. DFTB. Available online: http://www.dftb.org/ (accessed on 23 November 2015).

70. Gaus, M.; Goez, A.; Elstner, M. Parameterization and Benchmark of DFTB3 for Organic Molecules. J. Chem. Theory Comput. 2013, 9, 338-354.

71. Gaus, M.; Lu, X.; Elstner, M.; Cui, Q. Parameterization of DFTB3/3OB for sulfur and phosphorus for chemical and biological applications. J. Chem. Theory Comput. 2014, 10, 1518-1537.

72. Hehre, W.J.; Ditchfield, R.; Pople, J. Self-Consistent Molecular Orbital Methods. XII. Further Extensions of Gaussian-Type Basis Sets for Use in Molecular Orbital Studies of Organic Molecules. J. Chem. Phys. 1972, 56, 2257-2261.

73. Hariharan, P.C.; Pople, J.A. The influence of polarization functions on molecular orbital hydrogenation energies. Theor. Chem. Acc. (Theor. Chim. Acta) 1973, 28, 213-222.

74. Hehre, W.J.; Stewart, R.F.; Pople, J.A. Self-Consistent Molecular-Orbital Methods. I. Use of Gaussian Expansions of Slater-Type Atomic Orbitals. J. Chem. Phys. 1969, 51, 2657-2664.

75. Vosko, S.H.; Wilk, L.; Nusair, M. Accurate spin-dependent electron liquid correlation energies for local spin density calculations: A critical analysis. Can. J. Phys. 1980, 58, 1200-1211.

76. Becke3LYP Method References and General Citation Guidelines. Available online: http:// www.lct.jussieu.fr/manuels/Gaussian98/qa_sum94.htm (accessed on 30 November 2015).

77. Becke, A. Density-functional thermochemistry. III. The role of exact exchange. J. Chem. Phys. 1993, 98, 5648-5652.

78. Krishnan, R.; Binkley, J.S.; Seeger, R.; Pople, J.A. Self-consistent molecular orbital methods. XX. A basis set for correlated wave functions. J. Chem. Phys. 1980, 72, 650-654. 
79. Clark, T.; Chandrasekhar, J.; Schleyer, P.V.R. Efficient diffuse function-augmented basis sets for anion calculations. III. The 3-21+G basis set for first-row elements, Li-F. J. Comp. Chem. 1983, 4, 294-301.

80. Koval, P.; Foerster, D.; Sánchez-Portal, D. Fully self-consistent $G W$ and quasiparticle self-consistent $G W$ for molecules. Phys. Rev. B 2014, 89, 155417.

81. NIST. Available online: www.nist.gov (accessed on 30 November 2015)

82. CCCBDB. Available online: http://cccbdb.nist.gov/ (accessed on 30 November 2015)

83. Coulson, C.A. Present state of molecular structure calculations. Rev. Mod. Phys. 1960, 32, 170.

84. Pople, J.A. Two-dimensional chart of quantum chemistry. J. Chem. Phys. 1965, 43, S229-S230.

85. Karplus, M. Three-dimensional "Pople diagram". J. Phys. Chem. 1990, 94, 5436-5436.

86. Casida, M.E.; Salahub, D.R. Asymptotic correction approach to improving approximate exchange-correlation potentials: Time-dependent density-functional theory calculations of molecular excitation spectra. J. Chem. Phys. 2000, 113, 8918-8935.

87. Brundle, C.R.; Robin, M.B.; Basch, H. Electronic Energies and Electronic Structures of the Fluoromethanes. J. Chem. Phys. 1970, 53, 2196-2213.

88. Brundle, C.R.; Robin, M.B.; Basch, H.; Pinsky, M.; Bond, A. Experimental and theoretical comparison of the electronic structures of ethylene and diborane. J. Am. Chem. Soc. 1970, 92, 3863-3866.

89. Duffy, P.; Chong, D.P. Study of transition-state methods in the calculation of vertical ionization potentials by the local density approximation. Org. Mass. Spectr. 1993, 28, 321-326.

90. Takahashi, T.; Harada, Y.; Sato, N.; Seki, K.; Inokuchi, H.; Fujisawa, S. Gas and solid-phase photoelectron spectra of 5,6,11,12-tetraphenylnaphthacene (rubrene). Bull. Chem. Soc. Jpn. 1979, 52, 380-382.

91. D’Andrade, B.W.; Datta, S.; Forrest, S.R.; Djurovich, P.; Polikarpov, E.; Thompson, M.E. Relationship between the ionization and oxidation potentials of molecular organic semiconductors. Org. Electron. 2005, 6, 11-20.

92. Fitzner, R.; Reinold, E.; Mishra, A.; Mena-Osteritz, E.; Ziehlke, H.; Körner, C.; Leo, K.; Riede, M.; Weil, M.; Tsaryova, O.; et al. Dicyanovinyl-substituted oligothiophenes: Structure-property relationships and application in vacuum-processed small-molecule organic solar cells. Adv. Funct. Mater. 2011, 21, 897-910.

93. Berkowitz, J. Photoelectron spectroscopy of phthalocyanine vapors. J. Chem. Phys. 1979, 70, 2819-2828.

94. Piet, D.P.; Danovich, D.; Zuilof, H.; Sudhölter, E.J.R. Ionization potentials of porphyrins and phthalocyanines. A comparative benchmark study of fast improvements of Koopmans' theorem. J. Chem. Soc. Perkin Trans. 2 1999, 1999, 1653-1662.

95. Montalti, M.; Credit, A.; Prodi, L.; Gandolfi, M.T. Handbook of Photochemistry, 3rd ed.; Taylor and Francis: London, UK, 2006.

96. Tada, A.; Geng, Y.; Wei, Q.; Hashimoto, K.; Tajima, K. Tailoring organic heterojunction interfaces in bilayer polymer photovoltaic devices. Nat. Mater. 2011, 10, 450-455.

97. Sai, N.; Tiago, M.L.; Chelikowsky, J.R.; Reboredo, F.A. Optical spectra and exchange-correlation effects in molecular crystals. Phys. Rev. B 2008, 77, 161306(R). 
98. Wilke, A.; Endres, J.; Hörmann, U.; Niederhausen, J.; Schlesinger, R.; Frisch, J.; Amsalem, P.; Wagner, J.; Gruber, M.; Opitz, A.; et al. Correlation between interface energetics and open circuit voltage in organic photovoltaic cells. Appl. Phys. Lett. 2012, 101, 233301.

99. Cook, S.; Katoh, R.; Furube, A. Ultrafast Studies of Charge Generation in PCBM:P3HT Blend Films following Excitation of the Fullerene PCBM. J. Phys. Chem. C 2009, 113, 2547-2552.

100. Crocker, L.; Wang, T.B.; Kebarle, P. Electron affinities of some polycyclic aromatic hydrocarbons, obtained from electron-transfer equilibia. J. Am. Chem. Soc. 1993, 115, 7818-7822.

101. Huang, D.L.; Dau, P.D.; Liu, H.T.; Wang, L.S. High-resolution photoelectron imaging of cold $C_{60}^{-}$anions and accurate determination of the electron affinity of $C_{60}$. J. Chem. Phys. 2014, $140,224315$.

102. Akaike, K.; Kanai, K.; Yoshida, H.; Tsutsumi, J.; Nishi, T.; Sato, N.; Ouchi, Y.; Seki, K. Ultraviolet photoelectron spectroscopy and inverse photoemission spectroscopy of [6,6]-phenyl- $\mathrm{C}_{61}$-butyric acid methyl ester in gas and solid phases. J. Appl. Phys. 2008, 104, 023710.

103. Siegert, S.; Vogeler, F.; Marian, C.M.; Weinkauf, R. Throwing light on dark states of $\alpha$-oligothiophenes of chain lengths 2 to 6: radical anion photoelectron spectroscopy and excited-state theory. Phys. Chem. Chem. Phys. 2011, 13, 10350-10363.

104. Larson, B.W.; Whitaker, J.B.; Wang, X.; Popov, A.A.; Rumbles, G.; Kopidakis, N.; Strauss, S.H.; Boltalina, O.V. Electron Affinity of Phenyl- $\mathrm{C}_{61}$-Butyric Acid Methyl Ester $(P C B M)$. J. Phys. Chem. C 2013, 117, 14958-14964.

105. Tang, C.W. Two-layer organic photovoltaic cell. Appl. Phys. Lett. 1986, 48, 183.

106. Jain, S.C.; Willander, M.; Kumar, V. Conducting Organic Materials and Devices: Semiconductors and Semimetals; Academic Press: New York, NY, USA, 2007; Volume 81.

107. Singh, J. Semiconductor Devices: Basic Principles; John Wiley and Sons: New York, NY, USA, 2001.

108. Wagner, J.; Gruber, M.; Wilke, A.; Tanaka, Y.; Topczak, K.; Steindamm, A.; Hörmann, U.; Opitz, A.; Nakayama, Y.; Ishii, H.; et al. Identification of different origins for s-shaped current voltage characteristics in planar heterojunction solar cells. J. Appl. Phys. 2012, 111, 054509.

109. Huh, Y.H.; Park, B.; Hwang, I. Investigating the origin of S-shaped photocurrent-voltage characteristics of polymer:fullerene bulk-heterojunction organic solar cells. J. Appl. Phys. 2014, $115,124504$.

110. Shockley, W.; Queisser, H. Detailed balance limit of efficiency of $p-n$ junction solar cells. J. Appl. Phys. 1961, 32, 510-519.

111. Baruch, P.; Vos, A.D.; Landsberg, P.T.; Parrott, J.E. On some thermodynamics aspects of photovoltaic solar energy conversion. Sol. Energy Mater. Sol. Cells 1995, 36, 201-222.

112. Brabec, C.J.; Cravino, A.; Meissner, D.; Sariciftci, N.S.; Fromherz, T.; Rispens, M.; Sanchez, L.; Hummelen, J.C. Origin of the Open Circuit Voltage of Plastic Solar Cells. Adv. Funct. Mater. 2001, 11, 374-380.

113. Yu, G.; Pakbaz, K.; Heeger, A.J. Semiconducting polymer diodes: Large size, low cost photodetectors with excellent visible-ultraviolet sensitivity. Appl. Phys. Lett. 1994, $64,3422-3424$. 
114. Yu, G.; Heeger, A.J. Charge separation and photovoltaic conversion in polymer composites with internal donor/acceptor heterojunctions. J. Appl. Phys. 1995, 78, 4510-4515.

115. Mihailetchi, V.D.; Blom, P.W.M.; Hummelen, J.C.; Rispens, M.T. Cathode dependence of the open-circuit voltage of polymer:fullerene bulk heterojunction solar cells. J. Appl. Phys. 2003, 94, 6849-6854.

116. Scharber, M.C.; Mühlbacher, D.; Koppe, M.; Denk, P.; Waldauf, C.; Heeger, A.J.; Brabec, C.J. Design rules for donors in bulk-heterojunction solar cells-Towards $10 \%$ energy-conversion efficiency. Adv. Mater. 2006, 18, 789.

(c) 2015 by the authors; licensee MDPI, Basel, Switzerland. This article is an open access article distributed under the terms and conditions of the Creative Commons Attribution license (http://creativecommons.org/licenses/by/4.0/). 